\title{
Thermodynamic factors for locally non-neutral, concentrated electrolytic fluids
}

\author{
Priyamvada Goyal \\ Lawrence Berkeley National Laboratory, 1 Cyclotron Road, Berkeley, CA 94720, USA \\ Charles W. Monroe \\ Department of Engineering Science, University of Oxford, Parks Road, Oxford OX1 3PJ, UK \\ The Faraday Institution, Harwell Campus, Didcot OX11 ORA, UK
}

\begin{abstract}
A constitutive framework based on a novel two-ion secondary reference state is created to parameterize the compositiondependent parts of the thermodynamic differentials that undergird diffusion driving forces in non-neutral electrolytic fluids. A neutralizable composition basis is defined by replacing one natural composition variable with molar charge. This leads to a system of what we call core potentials, among which Maxwell relations and Gibbs-Duhem constraints take particularly simple forms. Matrix representations are constructed for transformations that interconvert among Guggenheim (electrochemical), Smyrl-Newman (quasielectrostatic), and core potentials. We extend the Guggenheim and Smyrl-Newman formalisms to charge-imbalanced microscopic equilibria and refine Kokotov's twin-potential scheme by using intensive descriptors of energy and composition. The framework can incorporate measurements of conventional electroneutral thermodynamic factors, but also includes new electrostatic colligative properties. Consistent thermodynamic parameterizations are derived for electrolytes obeying the ideal-solution approximation and electrolytes that follow Poisson-Boltzmann theory. We explicitly develop thermodynamically consistent expressions for species electrochemicalpotential differentials within a chemically nonideal solution of a symmetrical-valence electrolyte that also satisfies the Poisson-Boltzmann charge-potential relation.
\end{abstract}

\section{Background}

The implementation of transport simulations based on irreversible thermodynamics requires constitutive laws that express how diffusion driving forces depend on materialproperty gradients. Mass diffusion is driven primarily by chemical-potential gradients, so modelling requires parameters known as thermodynamic factors - colligative properties that quantify how activity changes with composition.

In electrolytic solutions, composition variation may be accompanied by changes in excess charge, whose capacity to perform electrical work also affects overall energetics. When thermodynamic laws are stated in the conventional way, information about a system's charge state is hard to isolate, because it is embedded in the set of species molar contents on which the free energy fundamentally depends. Moreover, the contribution of electrical work to system energy requires an explicit shift from chemical to electrochemical potentials.

The convolution of a system's chemical and electrical states presents two challenges that complicate the quantification of thermodynamic factors. First, one must address what we call Guggenheim's quandary [1]: since all

Email addresses: priyamvada@lbl.gov (Priyamvada Goyal), charles.monroe@eng.ox.ac.uk (Charles W. Monroe) pure chemical compounds are macroscopically electroneutral at equilibrium, the partial molar properties of charged species are intrinsically ambiguous. The measurement of a given ion's electrochemical potential (i.e., its partial molar free energy) in an electrolytic solution, for instance, demands that one vary the amount of that ion only, but this cannot be done because an electrolyte's ion content can only be changed in the laboratory by adding or removing neutral salts [2]. Second, one must determine whether the definition of electrical potential chosen for modelling purposes is experimentally accessible and thermodynamically meaningful $[3,4]$. It is concerning that no clearly defined experimental measurement process underpins the electric potentials used in widely adopted models such as Debye-Hückel theory [5], Poisson-Boltzmann analysis [68], Poisson-Nernst-Planck transport theory [9-12], and electrohydrodynamics [13-15], or in variational approaches based on Gibbs-energy functionals, deployed by Bazant and others [16-18].

Guggenheim himself [19], Smyrl and Newman [20], and, more recently, Kokotov [21] developed workarounds to circumvent Guggenheim's quandary and put electrical potential on a sound footing. When proposing the concept of electrochemical potential [19], Guggenheim introduced the notion of a secondary reference state, which relates one 
ion's electrochemical potential to the voltage measured by a heterogeneously equilibrated reference electrode reversible to that ion $[1,22,23]$. Smyrl and Newman deploy the secondary-reference-state idea more abstractly: they choose a particular charged species as a reference ion, whose electrostatic energy is proportional to a 'quasielectrostatic potential' and whose mixing free energy is that of a solute in an ideal solution; under conditions of local electroneutrality, the nonideal chemical potentials of all other charged species can be modelled in terms of the activities of neutral species formed in combination with the reference ion [20]. Kokotov goes further, circumventing the choice of a particular reference ion by exploiting the linear dependence of charged-species compositions within states at fixed charge. Fixing charge and sequentially eliminating each ion content in favor of the others leads to several equivalent statements of the Gibbs function, each with a different composition basis. Across these Gibbs functions, neutral combinations of electrochemical potentials, which Kokotov calls 'twin chemical potentials', emerge naturally as variables conjugate to composition. The twin chemical potentials are similar to Smyrl-Newman potentials, but have clearer connections to a measurement process, each being related to a partial Gibbs energy at fixed charge.

Given the recent surge of interest in electrochemical systems where nanoscale phenomena are not merely relevant but critically important, theoretical frameworks that can resolve regions of charge separation while maintaining thermodynamic rigor are much needed [24-32]. The recent parallel developments in experimental techniques, which now allow for direct observations of space charge domains, measurement of screening lengths, etc., promise new routes to develop and validate such frameworks [3336]. Molecular dynamics simulations have been used to explore species activity coefficients and potential definitions in non-electroneutral systems [37, 38], but similar efforts have largely been lacking in the continuum modelling field. Although existing Poisson-Nernst-Planck transport models [39-41] account for space charge domains, they neglect chemical non-idealities that originate in ion/ion interactions by assuming a limit of infinite dilution. There remains a need, therefore, for a thermodynamic description of concentrated electrolytes that remains consistent when local electroneutrality is violated [42]. It would be particularly useful to have a formalism with the generality of Kokotov's that is based on a clearly defined secondary reference state like Smyrl and Newman's, and which also relates easily to Guggenheim's standard electrochemical potentials.

\section{Approach}

Below we analyze the laws of classical thermodynamics to establish several relationships that constrain thermodynamic factors. By developing mathematical structures that derive from fundamental concepts such as the extensivity of energy, the convexity of free energy, and the
Maxwell relationships among material properties, and by taking Faraday's law to its logical conclusion, we produce a parsimonious and thermodynamically consistent constitutive framework for the thermodynamic factors in nonneutral, nonideal, multicomponent electrolytic solutions.

Drawing inspiration from both the Smyrl-Newman and Kokotov approaches, we outline a process by which, given two reference species that define a secondary reference state, changes of basis can be executed to deconvolute the effects of chemical composition from those of excess charge in elementary thermodynamic laws. The process hinges on two transformations: a change of composition basis, in which one of the independent particle fractions is replaced by a suitable charge quantifier; and a change of basis for the partial energies conjugate to composition, wherein one species' electrochemical potential is replaced by an appropriately defined electrical potential.

Certain choices of convention allow key operations to be stated as affine or linear transformations that depend only on the list of species charges. We construct matrix representations of these transformations and their inverses, facilitating straightforward mappings from the Guggenheim potentials into new sets of potentials - named neutralizable potentials and core potentials - whose electrical content is clearly distinguished. As well as accounting for electrical energy explicitly, these new potentials are parameterized directly in terms of colligative properties that can be measured at constant charge.

An identification of several new electrical thermodynamic properties closes the general development. An equation of state for molar charge is derived for an ideal electrolytic solution, and the thermodynamic factors are derived for a non-ideal electrolyte whose charge satisfies the Poisson-Boltzmann equation of state. To clarify the general procedures, we also work an example, establishing constitutive equations for species electrochemical potentials that are consistent with the Poisson-Boltzmann state equation for a chemically non-ideal binary solution of a symmetric salt in a neutral solvent.

\section{Preliminaries}

Fundamental thermodynamics. The derivation of OnsagerStefan-Maxwell transport equations relies on several laws from classical thermodynamics. Onsager's macroscopic analysis of liquid diffusion [43] implicitly assumes that the first law can be stated in a form that relates an intensive free-energy descriptor to changes in a set of thermodynamic intensive variables, and also assumes that an Euler equation governs this free energy. The Gibbs-Duhem relation implied by these two fundamental laws ultimately combines with a local, transient statement of the second law — a dissipation function — to identify the conjugate pairs of forces and fluxes associated with mass transport.

Here we state the fundamental relationships using definitions and notation laid out in our earlier paper on transient balance equations for solid electrolytes [44]. Within 
that framework, a phase's thermodynamics is based in its Gibbs free energy, $G$. For a system containing $n$ distinguishable chemical species, $G$ depends naturally on the system's temperature $T$ and the symmetric external stress tensor, as well as all the species' molar contents.

The present analysis is restricted to fluid electrolytes. By definition, fluids cannot sustain deformation stress at equilibrium. Consequently the stress is spherical and can be replaced by a scalar external pressure $p$. Constitutive laws for diffusion in solids require mechanical considerations that would take us too far afield, and will be addressed in a future paper.

Designate the molar content of species $i$ as $n_{i}$, and let $\left\{n_{i}\right\}_{n}$ indicate an ordered list of cardinality $n$ wherein the index of the bracketed quantity $-n_{i}$ in this case - ranges across all species from the first to the $n^{\text {th }}$. Classical thermodynamics begins with the assertion that some continuous, differentiable scalar function $G\left(T, p,\left\{n_{i}\right\}_{n}\right)$ quantifies the Gibbs free energy of a single $n$-ary fluid phase, and that all the quantities in the domain of this fundamental function can be varied independently.

The first law of thermodynamics for the systems of interest here is generally understood to be

$$
d G=-S d T+V d p+\sum_{i=1}^{n} \mu_{i} d n_{i},
$$

in which $S$ is the entropy, $V$ the volume, and $\mu_{i}$ the electrochemical potential of species $i$ defined by Guggenheim $[1,19]$. Pressure differentiation of equation 1 with temperature and all the molar contents fixed requires that

$$
V=\left(\frac{\partial G}{\partial p}\right)_{T, n_{i}}
$$

Thus a volume-explicit equation of state, $V\left(T, p,\left\{n_{i}\right\}_{n}\right)$, derives from the Gibbs function.

By introducing the total molar content of the fluid, $n_{\mathrm{T}}$,

$$
n_{\mathrm{T}}\left(\left\{n_{i}\right\}_{n}\right)=\sum_{i=1}^{n} n_{i}
$$

composition can alternatively be described by an $n$-tuple of particle fractions $\left\{y_{i}\right\}_{n}$, defined as

$$
y_{j}\left(\left\{n_{i}\right\}_{n}\right)=\frac{n_{j}}{n_{\mathrm{T}}\left(\left\{n_{i}\right\}_{n}\right)}
$$

for species $j$. In light of equation 3 , these composition descriptors must be constrained by the phase rule ${ }^{1}$

$$
\sum_{i=1}^{n} y_{i}=1, \quad \text { so that } \quad y_{n}\left(\left\{y_{i}\right\}_{n-1}\right)=1-\sum_{i=1}^{n-1} y_{i}
$$

\footnotetext{
${ }^{1}$ When placing properties of individual species in an $n$-tuple, ordinal numbers will always be assigned to the same species. In this way the notation $\left\{y_{i}\right\}_{n-1}$ produces a list wherein a particular species' particle fraction is removed from $\left\{y_{i}\right\}_{n}$, and the same species' molar content, say, is removed from $\left\{n_{i}\right\}_{n}$ by writing $\left\{n_{i}\right\}_{n-1}$.
}

which is to say, only $n-1$ of the particle fractions can be varied independently.

Rearrangement of equations 3 through 5 shows that

$$
n_{i}\left(\left\{y_{i}\right\}_{n-1}, n_{\mathrm{T}}\right)=\left\{\begin{array}{cc}
n_{\mathrm{T}} y_{i} & ; \quad i<n, \\
n_{\mathrm{T}}\left(1-\sum_{k=1}^{n-1} y_{k}\right) & ; \quad i=n .
\end{array}\right.
$$

Equations 4 and 6 establish an invertible transformation (bijective map), notated as $\left\{n_{i}\right\}_{n} \leftrightarrow\left\{\left\{y_{i}\right\}_{n-1}, n_{\mathrm{T}}\right\}$, between the basis of species molar contents and a basis comprising the independent particle fractions alongside total molar content. Thus it is equivalent to write the Gibbs energy as a function $G\left(T, p,\left\{y_{i}\right\}_{n-1}, n_{\mathrm{T}}\right)$.

The alternative formulation of composition makes $G$ depend on a single extensive state variable $n_{\mathrm{T}}$. In classical systems $G$ itself is taken to have the extensivity property $\lambda G=G\left(T, p,\left\{y_{i}\right\}_{n-1}, \lambda n_{\mathrm{T}}\right)$ for any $\lambda>0$. As a result, application of Euler's theorem to equation 1 shows that

$$
G\left(T, p,\left\{n_{i}\right\}_{n}\right)=\sum_{i=1}^{n} \mu_{i} n_{i}
$$

known as the Euler equation for $G$.

Intensive forms of the fundamental laws. The molar Gibbs energy $\bar{G}$, defined as $\bar{G}=\left(1 / n_{\mathrm{T}}\right) \cdot G$, depends entirely on intensive state variables, since extensivity of $G$ implies $\bar{G}=\bar{G}\left(T, p,\left\{y_{i}\right\}_{n-1}\right)$. Hereafter, unless stated otherwise, the term 'composition' refers only to the choice of $n-1$ independent intensive variables $\left\{y_{i}\right\}_{n-1}$, excluding $n_{\mathrm{T}}$.

Division of equation 7 through by $n_{\mathrm{T}}$ shows that one can write an Euler equation for molar Gibbs energy $\bar{G}$ as

$$
\bar{G}=\sum_{i=1}^{n} \mu_{i} y_{i}
$$

It follows that $\left\{\mu_{i}\right\}_{n}$ depends only on the intensive basis variables.

Since $V$ has the same domain and extensivity property as $G$, application of Euler's theorem to the differential expansion of $d V$ yields an equation of state for molar volume,

$$
\frac{1}{c_{\mathrm{T}}}=\sum_{i=1}^{n} \bar{V}_{i} y_{i}
$$

in which $c_{\mathrm{T}}=n_{\mathrm{T}} / V$ is the total molarity (inverse molar volume) and $\bar{V}_{i}$ is the partial molar volume of species $i$. All the state variables that appear in equations 8 and 9 have standard definitions in classical thermodynamics, given in terms of partial derivatives:

$$
\begin{aligned}
\mu_{i} & =\left(\frac{\partial G}{\partial n_{i}}\right)_{T, p, n_{j \neq i}} \text { and } \\
\bar{V}_{i} & =\left(\frac{\partial V}{\partial n_{i}}\right)_{T, p, n_{j \neq i}}=\left(\frac{\partial \mu_{i}}{\partial p}\right)_{T, n_{k}} .
\end{aligned}
$$

Partial molar volume $\bar{V}_{i}$ is primarily defined as the derivative of $V$ with molar content $n_{i}$; in light of equation 2 , 
pressure differentiation of equation 7 yields a Maxwell relation that also ties a species' partial molar volume to the pressure derivative of its electrochemical potential.

Manipulation of the preceding equations casts the first law in an intensive form (see Appendix A), as

$$
d \bar{G}=-\bar{S} d T+\frac{1}{c_{\mathrm{T}}} d p+\sum_{i=1}^{n-1}\left(\mu_{i}-\mu_{n}\right) d y_{i}
$$

Here the molar entropy $\bar{S}$ is defined in terms of partial molar entropies,

$$
\bar{S}=\sum_{i=1}^{n} \bar{S}_{i} y_{i}=-\sum_{i=1}^{n} y_{i}\left(\frac{\partial \mu_{i}}{\partial T}\right)_{p, n_{k}},
$$

wherein the last equality again delivers a Maxwell relation involving electrochemical potential. A total derivative of equation 8 combines with equation 1 to produce

$$
\sum_{i=1}^{n} y_{i} d \mu_{i}=-\bar{S} d T+\frac{1}{c_{\mathrm{T}}} d p
$$

This Gibbs-Duhem equation can be viewed as a statement of Newton's third law, which can be used to identify factors that drive mass transport.

Darken thermodynamic factors. Hirschfelder, Curtiss, and Bird manipulated the differential balances of mass, momentum, and energy to discover an expression for local energy dissipation [45]. After substituting equations 9 and 12 into equation 13 and rearranging, one finds that

$$
\sum_{i=1}^{n} y_{i}\left(d \mu_{i}+\bar{S}_{i} d T-\bar{V}_{i} d p\right)=0
$$

In the dissipation function derived by Hirschfelder et al., the $i^{\text {th }}$ term in this sum forms part of the vector conjugate to the molar excess flux of species $i$. Following de Groot's approach to irreversible thermodynamics, the $i^{\text {th }}$ term in equation 14 should therefore be taken to contribute part of the thermodynamic force that drives diffusion of species $i[46,47]^{2}$

The functionality of $\bar{G}$ requires that $\mu_{i}\left(T, p,\left\{y_{i}\right\}_{n-1}\right)$, so it follows by chain-rule expansion and the Maxwell relations from equations 10 and 12 that

$$
d \mu_{i}=\sum_{j=1}^{n-1} \frac{1}{y_{i}} \bar{Q}_{i j} d y_{j}-\bar{S}_{i} d T+\bar{V}_{i} d p
$$

where

$$
\bar{Q}_{i j}=y_{i}\left(\frac{\partial \mu_{i}}{\partial y_{j}}\right)_{T, p, y_{k \neq j, n}}
$$

\footnotetext{
${ }^{2}$ Formally this force drives mass diffusion; another part of the conjugate vector identified by Hirschfelder et al. drives pressure diffusion. The pressure-diffusion driving force is revealed by considering that species should not be driven to diffuse in homogeneous solutions at hydrostatic equilibrium [45]. Goyal and Monroe [44] present the complete driving force, which is not needed here.
}

Monroe and Newman defined a square matrix of thermodynamic factors $\mathbf{Q}$, whose entries are proportional to $\bar{Q}_{i j}$ for $i, j<n[48] .^{3}$ The $(n-1) \times(n-1)$ matrix composed of factors $\bar{Q}_{i j}$ is written here as $\overline{\mathbf{Q}}_{n-1}$; we call it the Darken matrix because it represents a multicomponent extension of the thermodynamic factor Darken originally developed to model binary diffusion in solids [49].

Even in systems where $T$ and $p$ change, the individual terms in equation 14 vary simply with composition. Rearrangement of equation 15 makes clear that the first $n-1$ terms in Gibbs-Duhem relation 14 can be denumerated columnwise by writing a matrix equation:

$$
\left[y_{i}\left(d \mu_{i}+\bar{S}_{i} d T-\bar{V}_{i} d p\right)\right]_{n-1}=\overline{\mathbf{Q}}_{n-1} d\left[y_{i}\right]_{n-1} .
$$

Here we have introduced the notation $\left[\alpha_{i}\right]_{n-1}$ to indicate an $(n-1) \times 1$ matrix - a column - whose $i^{\text {th }}$ row entry is $\alpha_{i} ; d\left[\alpha_{i}\right]_{n-1}$ stands for the total differential of a column, equivalent to $\left[d \alpha_{i}\right]_{n-1}$. Equation 17 is general for fluids, making concrete how thermodynamic-factor data determine the diffusion driving forces.

The particle-fraction Hessian. Equilibrated systems are stable to fluctuations, which imposes additional structure on the Gibbs function. The second law of thermodynamics mandates that $\bar{G}$ be minimal at equilibrium. Therefore, if a solution's composition is thermodynamically stable at a given composition, then $\bar{G}$ must be convex there. It is expected that every state accessed locally within a fluid undergoing simple diffusion is stable, so the function $\bar{G}$ should be convex at all accessible compositions.

Monroe, Wheeler, and Newman [50] defined a Hessian matrix, written here as a real matrix $\overline{\mathbf{K}}_{n-1},{ }^{4}$

$$
\left(\overline{\mathbf{K}}_{n-1}\right)_{i j}=\left(\frac{\partial^{2} \bar{G}}{\partial y_{i} \partial y_{j}}\right)_{T, p, y_{k \neq i, j, n}} .
$$

Because $\overline{\mathbf{K}}_{n-1}=\left(\overline{\mathbf{K}}_{n-1}\right)^{\mathrm{T}}$, the Hessian is diagonalizable by a rigid rotation and its eigenvalues are real. If $\bar{G}$ is convex, then $\overline{\mathbf{K}}_{n-1}$ is positive semidefinite throughout the accessible domain of $\left\{y_{i}\right\}_{n-1}$.

A direct connection exists between the Hessian matrix and the Darken matrix, substantiated as an equation involving a symmetric composition matrix $\mathbf{Y}_{n-1}$ [48]. Entries of both $\mathbf{Y}_{n-1}$ and its inverse can be written explicitly in indicial forms [51]:

$$
\left(\mathbf{Y}_{n-1}\right)_{i j}=\frac{\delta_{i j}}{y_{i}}+\frac{1}{y_{n}} \quad \text { and } \quad\left(\mathbf{Y}_{n-1}^{-1}\right)_{i j}=\delta_{i j} y_{j}-y_{i} y_{j}
$$

Here $\delta_{i j}$ is the Kronecker delta, equal to 1 if $i=j$ and 0 otherwise. The matrix determinant lemma shows that

$$
\operatorname{det}\left(\mathbf{Y}_{n-1}^{-1}\right)=\prod_{k=1}^{n} y_{k}
$$

\footnotetext{
${ }^{3}$ Monroe and Newman formed a matrix from $\bar{Q}_{i j} / R T$, in which $R$ is the gas constant; the definition in equation 16 simplifies notation.

${ }^{4}$ Monroe et al. used $\mathbf{K}=N_{\mathrm{A}} n_{\mathrm{T}} /(4 R T) \overline{\mathbf{K}}_{n-1}$, where $N_{\mathrm{A}}$ is Avogadro's number; we ignore the positive scalar prefactor for simplicity.
} 
so $\mathbf{Y}_{n-1}$ is invertible only when all $n$ species are present.

Sequential differentiation of equation 11 by $y_{i}$ and $y_{j}$ at fixed $T$ and $p$, followed by elimination of the partial derivatives of $\mu_{n}$ with equation 13 , gives

$$
\overline{\mathbf{K}}_{n-1}=\mathbf{Y}_{n-1} \overline{\mathbf{Q}}_{n-1} \text {. }
$$

Thus thermodynamic consistency, expressed through required properties of the Hessian matrix, imposes structure on the Darken matrix. In particular, equation 21 can be used to show that a similarity transformation with change of basis $\mathbf{Y}_{n-1}^{-1}$ sends the Darken matrix into its transpose: $\overline{\mathbf{Q}}_{n-1}^{\mathrm{T}}=\mathbf{Y}_{n-1} \overline{\mathbf{Q}}_{n-1} \mathbf{Y}_{n-1}^{-1}$. This conveniently shows how Maxwell relations constrain the entries of $\overline{\mathbf{Q}}_{n-1}$.

Outlook. Equations 8, 11, and 13 constitute the three foundational equilibrium relationships used in irreversible thermodynamics. Equation 17, constrained by the properties of the Hessian that appears in equation 21, provides the constitutive framework for diffusion driving forces that is needed to implement mass-transport models. The task before us is to show how variations in electrical state propagate through the fundamental governing equations, and to reveal the structures that underpin thermodynamic factors when charge is included explicitly as a state variable.

\section{Thermodynamics of electrolytic fluids}

Charge. Faraday's principle states that charge always resides on matter, and that a given chemical species will support its charge in a fixed amount that is independent of macroscopic temperature, stress, and composition. More formally, the excess charge $\mathrm{P}$ (units of coulombs) of an $n$ ary electrolytic system necessarily relates to its individual species contents through

$$
\mathrm{P}=F \sum_{i=1}^{n} z_{i} n_{i},
$$

in which $F$ is Faraday's constant and $z_{i}$ is the equivalent charge of species $i$. Division of both sides by the total number of moles allows this to be translated into an intensive quantity, the molar excess charge $\overline{\mathrm{P}}$, defined as

$$
\overline{\mathrm{P}}=F \sum_{i=1}^{n} z_{i} y_{i}
$$

Molar excess charge is convenient for thermodynamic analysis: being a linear function of the particle fractions alone, it is independent of temperature and stress. It can readily be used to compute the more familiar excess charge density $\rho_{\mathrm{e}}$,

$$
\rho_{\mathrm{e}}=c_{\mathrm{T}} \overline{\mathrm{P}},
$$

which is needed in the theory of electrodynamics to state a local form of Gauss's law. Because equation 23 requires that $\overline{\mathrm{P}}\left(\left\{y_{i}\right\}_{n-1}\right)$, this relationship reveals the temperature and stress dependences of $\rho_{\mathrm{e}}$ to be partitioned entirely into the volumetric equation of state, equation 9, ultimately determined by the $T$ and $p$ dependences of the $n$-tuple of partial molar volumes.
Neutralizable composition basis. To organize the analysis and ensure that all the transformations we propose remain mathematically consistent in every feasible electrochemical situation, we adopt the species-ordering convention of Monroe and Delacourt [52]. In a thermodynamically stable $n$-species electrolytic system, there must always be at least one species with positive charge and one with negative charge (because Gauss's law demands that charges balance globally in the equilibrium state). The Monroe-Delacourt convention takes species $n$ to be a charged species, called the reference ion, and species $n-1$ to be a countercharged species, henceforth called the charging ion. Selecting these two species identifies a two-ion secondary reference state.

Since the relationship between molar excess charge and the particle fractions is linear, it is straightforward to propose a new composition basis in which charge is associated with a single independent variable. Let the $(n-1)$-tuple $\left\{x_{i}\right\}_{n-1}$ represent a basis, which we will call neutralizable composition, in which the particle fraction of the charging ion is replaced by a (renormalized) molar charge density, so that $x_{n-1}=\overline{\mathrm{P}} /\left[F\left(z_{n}-z_{n-1}\right)\right]$.

The change of basis from independent particle fractions to neutralizable composition with respect to the two-ion secondary reference state is represented by an affine transformation $\left\{y_{i}\right\}_{n-1} \mapsto\left\{x_{i}\right\}_{n-1}$, given specifically as

$$
x_{i}=\left\{\begin{array}{cc}
y_{i} & ; \quad i<n-1, \\
\frac{z_{n}}{z_{n}-z_{n-1}}-\sum_{k=1}^{n-1}\left(\frac{z_{n}-z_{k}}{z_{n}-z_{n-1}}\right) y_{k} & ; \quad i=n-1 .
\end{array}\right.
$$

This transformation is affine, rather than linear, because it shifts the origin of the composition coordinates, as well as stretching and rotating them. Nevertheless, the basis change is invertible, and moreover, involutional; one can implement the inverse mapping $\left\{x_{i}\right\}_{n-1} \mapsto\left\{y_{i}\right\}_{n-1}$ just by swapping the positions of $x_{i}$ and $y_{i}$ in equation 25 . Since the transformation is bijective, $\left\{x_{i}\right\}_{n-1} \leftrightarrow\left\{y_{i}\right\}_{n-1}$, and therefore unique, either basis for composition in $\bar{G}$ is equally valid, and we may instead assume the functionality $\bar{G}\left(T, p,\left\{x_{i}\right\}_{n-1}\right)$ at the outset of thermodynamic analysis.

A linear transformation connects differential changes in the two composition bases. It is convenient to represent such transformations in matrix form: we write the two directions of the transformation $\left\{d y_{i}\right\}_{n-1} \leftrightarrow\left\{d x_{i}\right\}_{n-1}$ as

$$
\begin{aligned}
& d\left[x_{i}\right]_{n-1}=\mathbf{T}_{n-1}^{d x \leftrightarrow d y} d\left[y_{i}\right]_{n-1}, \\
& d\left[y_{i}\right]_{n-1}=\mathbf{T}_{n-1}^{d y \leftrightarrow d x} d\left[x_{i}\right]_{n-1} .
\end{aligned}
$$

Here the notation $\mathbf{T}_{n-1}^{d x \leftrightarrow d y}$ indicates the $(n-1) \times(n-1)$ matrix representation of the linear mapping from $\left\{d y_{i}\right\}_{n-1}$ into $\left\{d x_{i}\right\}_{n-1}$. As well as being compact, this way of expressing transformations between sets of variables helps to generalize results by induction, because the matrices can be cast in indicial forms. Here, for example,

$$
\left(\mathbf{T}_{n-1}^{d x \leftrightarrow d y}\right)_{i j}=\delta_{i j}-\delta_{i(n-1)}\left(\frac{z_{n}-z_{j}}{z_{n}-z_{n-1}}+\delta_{(n-1) j}\right) .
$$


The involutional nature of the transformation is demonstrated by the facts that $\mathbf{T}_{n-1}^{d x \leftrightarrow d y} \mathbf{T}_{n-1}^{d y \leftrightarrow d x}=\mathbf{I}_{n-1}$, where $\mathbf{I}_{n-1}$ is the $(n-1) \times(n-1)$ identity matrix, and that $\mathbf{T}_{n-1}^{d x \leftrightarrow d y}=$ $\mathbf{T}_{n-1}^{d y \leftrightarrow d x}$. Note also that since $\mathbf{T}_{n-1}^{d x \leftrightarrow d y}$ is lower triangular, with 1 s everywhere along the diagonal excepting a -1 in the $(n-1)^{\text {st }}$ position, it follows that $\operatorname{det}\left(\mathbf{T}_{n-1}^{d x \leftrightarrow d y}\right)=-1$.

Although the constraint in equation 5 shows that only $n-1$ particle fractions are independent, elementary relationships such as equations 8, 9, 12, 13, and 23 are more usefully expressed in terms of sums that range over all species. To facilitate injecting the neutralizable composition basis into equations such as these, it will be convenient to define an $n^{\text {th }}$ member of that set, $x_{n}$, equal to $y_{n}$. Appendix $\mathrm{B}$ shows that with this definition, there exists a bijective mapping $\left\{y_{i}\right\}_{n} \leftrightarrow\left\{x_{i}\right\}_{n}$ that can be represented as a matrix multiplication.

Electric potential. As well as being expressed by the composition basis, electrical information is also embedded in the set of electrochemical potentials. To isolate electrical energetics, the basis of these potentials must also be changed, requiring that we consider how to define the electric potential. Several options exist already, including the Nernst-Planck potential used in dilute-solution theory [53]; the reference-electrode potential used in Newman's concentrated-solution thermodynamics [22]; or the SmyrlNewman quasielectrostatic potential [20]. None of these definitions is rooted in electrostatics, however.

In physics, the electric potential is commonly understood to express how changes in charge affect changes in energy, i.e., potential is seen as the intensive thermodynamic variable conjugate to charge. Isolation of electrical energy from the other forms of energy embedded in the Gibbs function requires charge content to be varied without changing temperature, stress, or composition.

Of course, in an electrolytic system, composition cannot be fixed entirely if charge is to vary, demanding some clarification of the measurement process for the thermodynamic potentials. To describe this concisely, we shall call $\left\{y_{i}\right\}_{n-2}$ (or $\left\{x_{i}\right\}_{n-2}$ ) the chemical composition variables, and $\overline{\mathrm{P}}$ (or $x_{n-1}$ ) the electrical state variable.

Thermodynamic potentials can be established generally by a two-step process, in which the chemical composition and the electrical state are separately perturbed. The potentials conjugate to the chemical state can be measured by individually varying each of the species particle fractions, excluding those of the charging ion and reference ion: the set $\left\{y_{i}\right\}_{n-2}$ can be varied freely - observing the constraints that $y_{i}>0$ and $\sum_{i=1}^{n-2} y_{i}<1$, of course - without varying the system's charge. The electrical potential is determined by varying the electrical state, which can generally be done by changing the balance of the reference ion and charging ion while holding the chemical composition constant. Note that changes associated with these state variations are path independent, i.e., the change in Gibbs energy when moving from one overall state to another will be the same, regardless of the order in which one varies the chemical composition and the electrical state.

Mathematically, the energy change due to a reversible change in electrical state corresponds to a thermodynamic derivative, thus identifying what we shall call the naive electric potential $\Phi$, as

$$
\Phi=\left(\frac{\partial \bar{G}}{\partial \overline{\mathrm{P}}}\right)_{T, p, y_{i<n-1}} .
$$

This can be understood more concretely by changing the composition basis in the first law. Start with equation 11, replace $\left\{d y_{i}\right\}_{n-1}$ with $\left\{d x_{i}\right\}_{n-1}$ by using the transformation $\mathbf{T}_{n-1}^{d y \leftrightarrow d x}$ (cf. equation 27) under the summation, then differentiate with $x_{n-1}$ to show that

$$
\Phi=\frac{1}{F\left(z_{n}-z_{n-1}\right)}\left(\frac{\partial \bar{G}}{\partial x_{n-1}}\right)_{T, p, x_{i<n-1}}=\frac{\mu_{n}-\mu_{n-1}}{F\left(z_{n}-z_{n-1}\right)} .
$$

The naive electric potential quantifies the electrochemical potential difference between the reference and charging ions, normalized by the difference in their molar charges.

One can isolate electrical behavior within the thermodynamic laws by introducing the naive electric potential and expressing composition in terms of the neutralizable basis. In equation 8 , change the composition basis from $\left\{y_{i}\right\}_{n}$ to $\left\{x_{i}\right\}_{n}$ using $\mathbf{T}_{n}^{y \leftrightarrow x}$ from Appendix B, then eliminate the electrochemical potential of the reference ion in favor of $\Phi$ with equation 29. Back-substitution of the definitions from equation 25 then shows that

$$
\begin{aligned}
\bar{G}= & \sum_{i=1}^{n-2}\left[\left(\mu_{i}-F z_{i} \Phi\right)-\left(\mu_{n-1}-F z_{n-1} \Phi\right)\right] y_{i} \\
& +\Phi \overline{\mathrm{P}}+\left(\mu_{n-1}-F z_{n-1} \Phi\right) .
\end{aligned}
$$

Within this alternative statement of Euler's equation, the contribution that charge makes to the total free energy, $\Phi \overline{\mathrm{P}}$, stands alone.

Observe that groups of terms in equation 30 relate to quantities defined in other constitutive frameworks. For any species $i$, one can use equation 29 to show that

$$
\mu_{i}-F z_{i} \Phi=\left(\mu_{i}-\frac{z_{i}}{z_{n}} \mu_{n}\right)+\frac{z_{i}}{z_{n}-z_{n-1}}\left(\mu_{n-1}-\frac{z_{n-1}}{z_{n}} \mu_{n}\right) ;
$$

as well as being a linear combination of Guggenheim potentials, $\mu_{i}-F z_{i} \Phi$ is a weighted sum of the Smyrl-Newman chemical potential of ion $i$ (the first term in parentheses) and the Smyrl-Newman potential of the charging ion (the second). Set $i=n-1$ or $i=n$ here to find

$$
\begin{aligned}
\mu_{n-1}-F z_{n-1} \Phi & =\frac{z_{n}}{z_{n}-z_{n-1}}\left(\mu_{n-1}-\frac{z_{n-1}}{z_{n}} \mu_{n}\right) \\
& =\frac{\left[z_{n} \mu_{n-1}-z_{n-1} \mu_{n}\right]}{z_{n}-z_{n-1}} .
\end{aligned}
$$

Thus $\mu_{n-1}-F z_{n-1} \Phi$ is proportional to both the SmyrlNewman chemical potential of the charging ion (in parentheses) and the Kokotov twin chemical potential of the reference-ion/charging-ion pair (in square brackets). 
Issues with non-neutrality. Many existing frameworks lose thermodynamic consistency when applied to non-neutral systems. Analyses based on Guggenheim, Poisson-NernstPlanck, or Smyrl-Newman potentials usually employ constitutive laws that parse the electrochemical potential of every species into distinct chemical and electrical parts,

$$
\mu_{i}=\mu_{i}^{\text {chem }}+F z_{i} \Phi
$$

where $\mu_{i}^{\text {chem }}$ is taken to be independent of $\Phi$. Application of such laws to non-neutral systems poses two essential problems. First, insertion of equation 33 into the (isothermal, isobaric) Gibbs-Duhem equation, ${ }^{5}$ followed by some rearrangement with equations 9 and 12, yields

$$
\sum_{i=1}^{n} y_{i} d \mu_{i}^{\mathrm{chem}}=-\overline{\mathrm{P}} d \Phi
$$

If $d \mu_{i}^{\text {chem }}=R T d \ln \left(c_{\mathrm{T}} y_{i}\right)$ for each species, as assumed in typical Nernst-Planck models, then the left side of this Gibbs-Duhem equation vanishes, leading to a contradiction. (The supposed $\Phi$ independence of $\mu_{i}^{\text {chem }}$ demands that $c_{\mathrm{T}}$ be independent of $\Phi$.) If $\mu_{i}^{\text {chem }}$ is defined as it is in Smyrl-Newman theory, then the left side of equation 34 also vanishes, owing to how the ions' activity coefficients are constructed. Second, and more straightforwardly, the definition from equation 29 can be used to show that

$$
\mu_{n-1}-F z_{n-1} \Phi=\mu_{n}-F z_{n} \Phi .
$$

Thus, one cannot simply write equation 33 as a constitutive law for every species' electrochemical potential, because that form imposes an additional constraint on the potentials of the charging and reference ion that is separate from the Gibbs-Duhem equation.

It is important to recognize that the electric potential within a system is a thermodynamic property whose value stems from a relationship among the electrochemical potentials of the system's constituent species. Therefore, when one introduces an electric descriptor such as the naive potential $\Phi$, one must discard one of the species electrochemical potentials for which one writes compositiondependent constitutive laws.

Neutralizable electrochemical potentials. Inspection of equation 30 nevertheless suggests the definition of a new basis of conjugate thermodynamic potentials, which can be used in place of $\left\{\mu_{i}\right\}_{n}$. Define the neutralizable electrochemical potential of species $i, \mathrm{M}_{i}$, as

$$
\mathrm{M}_{i}=\left\{\begin{array}{cc}
\mu_{i}-F z_{i} \Phi & ; \quad i \neq n-1 \\
\mu_{n}-\mu_{n-1}=F\left(z_{n}-z_{n-1}\right) \Phi & ; \quad i=n-1
\end{array}\right.
$$

for $i \in\{1, \ldots, n\}$, a basis in which the naive electric potential replaces the charging ion's electrochemical potential,

\footnotetext{
${ }^{5} \mathrm{~A}$ similar conclusion can be drawn in the non-isothermal, nonisobaric case; we assume constant $T$ and $p$ for brevity.
}

and the remaining electrochemical potentials are replaced with forms that look like the 'chemical parts' of electrochemical potentials from prior theories.

Through equation 31, one can construct mappings that interconvert the Guggenheim potential basis $\left\{\mu_{i}\right\}_{n}$ and the neutralizable potentials $\left\{\mathrm{M}_{i}\right\}_{n}$. The mappings are linear, with matrix representations

$$
\left[\mathrm{M}_{i}\right]_{n}=\mathbf{T}_{n}^{\mathrm{M} \leftrightarrow \mu}\left[\mu_{i}\right]_{n} \quad \text { or } \quad\left[\mu_{i}\right]_{n}=\mathbf{T}_{n}^{\mu \leftrightarrow \mathrm{M}}\left[\mathrm{M}_{i}\right]_{n},
$$

and both directions of the transformation can be put in explicit indicial forms:

$$
\begin{gathered}
\left(\mathbf{T}_{n}^{\mathrm{M} \leftrightarrow \mu}\right)_{i j}=\delta_{i j}\left(1-2 \delta_{j(n-1)}\right)+\delta_{i(n-1)} \delta_{n j} \\
+\frac{z_{i}\left(1-\delta_{i(n-1)}\right)\left(\delta_{(n-1) j}-\delta_{n j}\right)}{z_{n}-z_{n-1}}, \\
\left(\mathbf{T}_{n}^{\mu \leftrightarrow \mathrm{M}}\right)_{i j}=\delta_{i j}+\delta_{i(n-1)}\left(\delta_{n j}-\delta_{(n-1) j}\right)+\frac{z_{i} \delta_{(n-1) j}}{z_{n}-z_{n-1}} .
\end{gathered}
$$

Direct computation shows that $\mathbf{T}_{n}^{\mathrm{M} \leftrightarrow \mu} \mathbf{T}_{n}^{\mu \leftrightarrow \mathrm{M}}=\mathbf{I}_{n}$, so the mapping is a bijection, $\left\{\mu_{i}\right\}_{n} \leftrightarrow\left\{\mathrm{M}_{i}\right\}_{n}$.

In terms of $\left\{\mathrm{M}_{i}\right\}_{n}$, the Euler equation for molar Gibbs energy becomes

$$
\begin{aligned}
\bar{G} & =\sum_{i=1}^{n-2} \mathrm{M}_{i} x_{i}+\mathrm{M}_{n}\left(1-\sum_{i=1}^{n-2} x_{i}\right)+\mathrm{M}_{n-1} x_{n-1} \\
& =\sum_{i=1}^{n-2} \mathrm{M}_{i} y_{i}+\mathrm{M}_{n}\left(1-\sum_{i=1}^{n-2} y_{i}\right)+\Phi \overline{\mathrm{P}}
\end{aligned}
$$

and the first law of thermodynamics transforms to

$$
\begin{aligned}
d \bar{G}= & -\bar{S} d T+\frac{1}{c_{\mathrm{T}}} d p+\sum_{i=1}^{n-2}\left(\mathrm{M}_{i}-\mathrm{M}_{n}\right) d x_{i} \\
& +\mathrm{M}_{n-1} d x_{n-1} \\
= & -\bar{S} d T+\frac{1}{c_{\mathrm{T}}} d p+\sum_{i=1}^{n-2}\left(\mathrm{M}_{i}-\mathrm{M}_{n}\right) d y_{i}+\Phi d \overline{\mathrm{P}}
\end{aligned}
$$

Equations 40 and 41 can be combined to produce

$$
\sum_{i=1}^{n-2} y_{i} d \mathrm{M}_{i}+\left(1-\sum_{i=1}^{n-2} y_{i}\right) d \mathrm{M}_{n}=-\overline{\mathrm{P}} d \Phi-\bar{S} d T+\frac{1}{c_{\mathrm{T}}} d p
$$

a transformed Gibbs-Duhem relation.

This formulation of the thermodynamic laws is appealing because it reduces to the standard structure from nonelectrochemical thermodynamics when $\overline{\mathrm{P}}=0\left(x_{n-1}=0\right)$. In that case the number of independent composition variables will be $n-2$, because electroneutrality and the phase rule combine to show that $y_{n-1}\left(\left\{y_{i}\right\}_{n-2}\right)$, a constraint that allows the charging ion's particle fraction to be struck from the composition basis.

Although local electroneutrality constrains the composition, incorporating the assumption $\overline{\mathrm{P}}=0$ into equations 40 through 42 shows that the naive potential disappears from all three. This $\Phi$ independence of the thermodynamic laws allows for variations in how potential is defined - and ensures that many of the different conventions used historically are thermodynamically consistent for electroneutral systems. Specifically, electric potentials defined within Nernst-Planck transport models [9], 
Guggenheim's electrochemical thermodynamics [1, 54], or Newman's concentrated-solution theory [22] all have equal merit under electroneutrality, although the latter two relate more concretely to experimental voltages [53].

Core potentials. An electrolyte's response to changes in its chemical composition or electrical state is constrained in more ways, through mathematical properties of the Gibbs function. To summarize these constraints concisely, it will be useful to introduce an additional basis change, which removes the differences between neutralizable potentials from equations 41 and 42 . Define the potential $\mathrm{M}_{i}^{n}$ as

$$
\mathrm{M}_{i}^{n}=\left\{\begin{array}{ccc}
\mathrm{M}_{i}-\mathrm{M}_{n}=\mu_{i}-\frac{z_{n}-z_{i}}{z_{n}-z_{n-1}} \mu_{n-1} & ; & i<n-1, \\
-\frac{z_{i}-z_{n-1}}{z_{n}-z_{n-1}} \mu_{n} & & \\
\mathrm{M}_{n-1}=F\left(z_{n}-z_{n-1}\right) \Phi=\mu_{n}-\mu_{n-1} & ; & i=n-1, \\
\mathrm{M}_{n}=\frac{z_{n} \mu_{n-1}-z_{n-1} \mu_{n}}{z_{n}-z_{n-1}} & ; & i=n .
\end{array}\right.
$$

We refer to $\left\{\mathrm{M}_{i}^{n}\right\}_{n}$ as the set of core potentials over the two-ion secondary reference state.

Changes of basis $\left\{\mathrm{M}_{i}\right\}_{n} \leftrightarrow\left\{\mathrm{M}_{i}^{n}\right\}_{n}$ can be implemented in the forward and backward directions by matrices

$$
\begin{aligned}
& \left(\mathbf{T}_{n}^{\mathrm{M}^{n} \leftrightarrow \mathrm{M}}\right)_{i j}=\delta_{i j}-\left(1-\delta_{i(n-1)}-\delta_{i n}\right) \delta_{n j}, \\
& \left(\mathbf{T}_{n}^{\mathrm{M} \leftrightarrow \mathrm{M}^{n}}\right)_{i j}=\delta_{i j}+\left(1-\delta_{i(n-1)}-\delta_{i n}\right) \delta_{n j} .
\end{aligned}
$$

A basis change with $\mathbf{T}_{n}^{\mathrm{M}^{n} \leftarrow \mathrm{M}}$ recasts the first law as

$$
\begin{aligned}
d \bar{G} & =-\bar{S} d T+\frac{1}{c_{\mathrm{T}}} d p+\sum_{i=1}^{n-1} \mathrm{M}_{i}^{n} d x_{i} \\
& =-\bar{S} d T+\frac{1}{c_{\mathrm{T}}} d p+\sum_{i=1}^{n-2} \mathrm{M}_{i}^{n} d y_{i}+\Phi d \overline{\mathrm{P}} .
\end{aligned}
$$

The first form shows that the core potentials are naturally conjugate to the neutralizable basis $\left\{x_{i}\right\}_{n-1}$. The second includes the naive potential to show that $\mathrm{M}_{n-1}^{n}$ is conjugate to the electrical state, whereas the subset $\left\{\mathrm{M}_{i}^{n}\right\}_{n-2}$ is conjugate to chemical composition. We call $\left\{\mathrm{M}_{i}^{n}\right\}_{n-2}$ core chemical potentials to emphasize the latter conjugacy.

In terms of core potentials, the Euler equation becomes

$$
\begin{aligned}
\bar{G} & =\sum_{i=1}^{n-1} \mathrm{M}_{i}^{n} x_{i}+\mathrm{M}_{n}^{n} \\
& =\sum_{i=1}^{n-2} \mathrm{M}_{i}^{n} y_{i}+\Phi \overline{\mathrm{P}}+\mathrm{M}_{n}^{n} .
\end{aligned}
$$

Transforming equation 42 produces

$$
\begin{aligned}
d \mathrm{M}_{n}^{n} & =-\sum_{i=1}^{n-1} x_{i} d \mathrm{M}_{i}^{n}-\bar{S} d T+\frac{1}{c_{\mathrm{T}}} d p \\
& =-\sum_{i=1}^{n-2} y_{i} d \mathrm{M}_{i}^{n}-\overline{\mathrm{P}} d \Phi-\bar{S} d T+\frac{1}{c_{\mathrm{T}}} d p
\end{aligned}
$$

a Gibbs-Duhem relation among core potentials.
Sequential application of transformations from equations 38 and 44 shows that the matrix with entries

$$
\begin{aligned}
\left(\mathbf{T}_{n}^{\mathrm{M}^{n} \leftrightarrow \mu}\right)_{i j}= & \delta_{i j}-\delta_{i(n-1)} \delta_{(n-1) j}+\delta_{i(n-1)} \delta_{n j} \\
& -\left(\frac{z_{n}-z_{i}}{z_{n}-z_{n-1}}-\frac{z_{n}}{z_{n}-z_{n-1}} \delta_{i n}\right) \delta_{(n-1) j} \\
& -\left(\frac{z_{i}-z_{n-1}}{z_{n}-z_{n-1}}+\frac{z_{n-1}}{z_{n}-z_{n-1}} \delta_{i n}\right) \delta_{n j}
\end{aligned}
$$

transforms Guggenheim electrochemical potentials directly into core potentials. Similarly, equations 45 and 39 yield

$$
\begin{aligned}
\left(\mathbf{T}_{n}^{\mu \leftrightarrow \mathrm{M}^{n}}\right)_{i j}= & \delta_{i j}+\left(\frac{z_{i}}{z_{n}-z_{n-1}}-\delta_{i(n-1)}\right) \delta_{(n-1) j} \\
& +\left(1-\delta_{i n}\right) \delta_{n j}
\end{aligned}
$$

as entries of the inverse transformation.

Equation 46 reveals that the core chemical potentials $\left\{\mathrm{M}_{i}^{n}\right\}_{n-2}$ identify with partial derivatives of molar energy with respect to chemical composition at fixed temperature, pressure, and electrical state:

$$
\mathrm{M}_{i}^{n}=\left(\frac{\partial \bar{G}}{\partial y_{i}}\right)_{T, p, y_{k<n-1, k \neq i}, \overline{\mathrm{P}}} \text { if } i<n-1 .
$$

These properties, based on a derivative similar to the one Kokotov used to find twin potentials [21], can be measured experimentally without encountering Guggenheim's quandary.

By differentiating the energy twice, one can also derive Maxwell relations involving the chemical composition:

$$
\left(\frac{\partial \mathrm{M}_{i}^{n}}{\partial y_{j}}\right)_{T, p, y_{k<n-1, k \neq j}, \overline{\mathrm{P}}}=\left(\frac{\partial \mathrm{M}_{j}^{n}}{\partial y_{i}}\right)_{T, p, y_{k<n-1, k \neq i}, \overline{\mathrm{P}}} ; i, j<n-1 .
$$

This is trivial when $n=2$ or 3 , but when $n>3$ it produces a distinct constraint for every meaningful combination of positive integers $i$ and $j$ such that $i<n-1$ and $j<i$, amounting to $(n-2)(n-3) / 2$ Maxwell relations among the core chemical potentials.

For any electrolyte comprising more than two species, one can also write $n-2$ Maxwell relations that connect changes in the naive electrical potential with changes in the chemical potentials,

$$
\left(\frac{\partial \Phi}{\partial y_{i}}\right)_{T, p, y_{k<n-1, k \neq i}, \overline{\mathrm{P}}}=\left(\frac{\partial \mathrm{M}_{i}^{n}}{\partial \overline{\mathrm{P}}}\right)_{T, p, y_{k<n-1}} \text { if } i<n-1 .
$$

Together, equations 52 and 53 produce $(n-1)(n-2) / 2$ Maxwell relations that must be satisfied by a thermodynamically consistent set of constitutive laws.

\section{Material properties for electrolytes}

We are now in a position to define a new set of material properties for electrolytic fluids, which quantify how the core potentials vary with respect to neutralizable composition over the two-ion secondary reference state. These new thermodynamic factors will be shown to relate in a 
straightforward way to the Darken matrix, allowing them to be used directly in constitutive laws for diffusion driving forces that rely on equation 17. Additional structures are also produced to develop equations of state for volume and entropy that cast partial molar volumes in terms of quantities that can be measured over a composition basis that allows charge to be held constant.

Core thermodynamic factors. The core potentials can be used to build a set of thermodynamic factors similar to the Darken matrix $\overline{\mathbf{Q}}_{n-1}$. Define the core thermodynamic factor $\overline{\mathrm{X}}_{i j}$ as the partial derivative of the $i^{\text {th }}$ core potential with respect to the $j^{\text {th }}$ neutralizable composition variable,

$$
\overline{\mathrm{X}}_{i j}=\left(\frac{\partial \mathrm{M}_{i}^{n}}{\partial x_{j}}\right)_{T, p, x_{k \neq j, k<n}} .
$$

Here $i$ ranges from 1 to $n$ and $j$ from 1 to $n-1$, so this definition establishes $n(n-1)$ properties. Not all of these properties are independent, however. Because differentiation of Gibbs-Duhem relation 48 with $x_{j}$ shows that

$$
\overline{\mathrm{X}}_{n j}=-\sum_{i=1}^{n-1} x_{i} \overline{\mathrm{X}}_{i j}
$$

it suffices to define only $(n-1)^{2}$ properties. Introduce the square matrix $\overline{\mathbf{X}}_{n-1}$ such that

$$
\left(\overline{\mathbf{X}}_{n-1}\right)_{i j}=\left(\frac{\partial \mathrm{M}_{i}^{n}}{\partial x_{j}}\right)_{T, p, x_{k \neq j, k<n}}=\overline{\mathrm{X}}_{i j} ; i, j<n .
$$

One can then enforce all $(n-1)(n-2) / 2$ of the Maxwell relations expressed by equations 52 and 53 by imposing a matrix symmetry:

$$
\overline{\mathbf{X}}_{n-1}=\overline{\mathbf{X}}_{n-1}^{\mathrm{T}} \text {. }
$$

Symmetry guarantees that $\overline{\mathbf{X}}_{n-1}$ has real eigenvalues and is diagonalizable by rigidly rotating the composition basis.

Equations 46 and 56 also demonstrate that $\overline{\mathbf{X}}_{n-1}$ is a Hessian of $\bar{G}$ like the previously defined matrix $\overline{\mathbf{K}}_{n-1}$, but expressed over the neutralizable composition basis:

$$
\left(\overline{\mathbf{X}}_{n-1}\right)_{i j}=\left(\frac{\partial^{2} \bar{G}}{\partial x_{i} \partial x_{j}}\right)_{T, p, x_{k \neq i, j, n}}
$$

Because $\left\{x_{i}\right\}_{n} \leftrightarrow\left\{y_{i}\right\}_{n}$ and the transformation $\mathbf{T}_{n-1}^{d x \leftrightarrow d y}$ has constant entries, a direct chain-rule expansion of the function $\bar{G}\left(T, p,\left\{y_{i}\left(\left\{x_{i}\right\}_{n-1}\right)\right\}_{n-1}\right)$ shows that

$$
\overline{\mathbf{K}}_{n-1}=\left(\mathbf{T}_{n-1}^{d x \leftrightarrow d y}\right)^{\mathrm{T}} \overline{\mathbf{X}}_{n-1} \mathbf{T}_{n-1}^{d x \leftrightarrow d y}
$$

thus the two Hessians are congruent. Congruent matrices have equal signatures [55]: $\overline{\mathbf{X}}_{n-1}$ is positive semidefinite whenever $\overline{\mathbf{K}}_{n-1}$ is; and if either matrix has a null space, the other has one of equal dimension. Note also that equation 59 is compatible with the symmetries of both Hessian matrices implied by Maxwell relations.
Use of equation 21 to eliminate $\overline{\mathbf{K}}_{n-1}$ from equation 59 expresses the Darken matrix in terms of the core thermodynamic factors, as

$$
\overline{\mathbf{Q}}_{n-1}=\mathbf{Y}_{n-1}^{-1}\left(\mathbf{T}_{n-1}^{d x \leftrightarrow d y}\right)^{\mathrm{T}} \overline{\mathbf{X}}_{n-1} \mathbf{T}_{n-1}^{d x \leftrightarrow d y}
$$

Thus the set of $(n-1)^{2}$ Darken factors depends explicitly on composition, species charges, and a set of $\frac{1}{2}(n-1)(n-2)$ material properties that constitute independent entries of the real, symmetric, positive semidefinite matrix $\overline{\mathbf{X}}_{n-1}$.

Equation 60 is the first major result of this analysis. It relates the standard Darken matrix, based on particlefraction derivatives of a set of independently variable electrochemical potentials, to a smaller set of material properties embodied by core thermodynamic factors over a twoion secondary reference state.

All the terms that express how species activities and electric potential vary with excess charge reside in the $(n-1)^{\text {st }}$ row of $\overline{\mathbf{X}}_{n-1}$, and the electric potential's composition dependence resides in the $(n-1)^{\text {st }}$ column, thereby distinguishing electrical effects. The symmetry expressed by equation 57 implies that $\overline{\mathbf{X}}_{n-1}$ can be parameterized in terms of its lower-triangular entries alone: the matrix contains $\frac{1}{2} n(n-1)$ independent material properties, dependent on temperature, pressure, and neutralizable composition.

Gibbs-Duhem extensions. Putting equations 17 and 60 together suffices to parameterize diffusion driving forces in a general and parsimonious way - in terms of the $T, p$, and $\left\{x_{i}\right\}_{n-1}$ dependences of the lower-triangular entries in the property matrix $\overline{\mathbf{X}}_{n-1}$. It is often convenient to work with the electrochemical potentials of all $n$ constituent species, however. It will be useful to formulate a property matrix that contains all $n(n-1)$ of the thermodynamic factors defined by equation 54, but which also clearly represents the true number of parameters that remain free after considering Maxwell and Gibbs-Duhem relations.

Introduce the symbol $\overline{\mathbf{X}}$ to represent the $n \times(n-1)$ matrix built up of all the core thermodynamic factors $\overline{\mathrm{X}}_{i j}$ defined by equation 54 . The thermodynamic constraints among the $n(n-1)$ properties that make up $\overline{\mathbf{X}}$ can be made explicit by expressing $\overline{\mathbf{X}}$ as a matrix product,

$$
\overline{\mathbf{X}}=\boldsymbol{\Gamma}_{x} \overline{\mathbf{X}}_{n-1},
$$

in which the $n \times(n-1)$ matrix $\boldsymbol{\Gamma}_{x}\left(\left\{x_{i}\right\}_{n-1}\right)$ is a function of the neutralizable composition basis, with entries

$$
\left(\boldsymbol{\Gamma}_{x}\right)_{i j}=\delta_{i j}-\delta_{i n} x_{j}
$$

We shall call the transformation in equation 61 a GibbsDuhem extension of symmetric core properties to form the complete set of core thermodynamic factors.

A similar process extends equation 17. Insertion of equation 15 into equation 14 and rearrangement produce

$$
\sum_{j=1}^{n-1}\left(\bar{Q}_{n j}+\sum_{i=1}^{n-1} \bar{Q}_{i j}\right) d y_{j}=0
$$


linear independence requires that the reference ion's Darken factors satisfy $\bar{Q}_{n j}=-\sum_{i=1}^{n-1} \bar{Q}_{i j}$. One can therefore write a Gibbs-Duhem extension of equation 17 as

$$
\left[y_{i}\left(d \mu_{i}+\bar{S}_{i} d T-\bar{V}_{i} d p\right)\right]_{n}=\overline{\mathbf{Q}} d\left[y_{i}\right]_{n-1},
$$

in which the $n \times(n-1)$ extended Darken matrix $\overline{\mathbf{Q}}$ is defined as

$$
\overline{\mathbf{Q}}=\boldsymbol{\Gamma}_{1} \overline{\mathbf{Q}}_{n-1},
$$

and the $n \times(n-1)$ extension matrix $\boldsymbol{\Gamma}_{1}$ has entries

$$
\left(\boldsymbol{\Gamma}_{1}\right)_{i j}=\delta_{i j}-\delta_{i n}
$$

Equation 64 augments equation 17 to include the thermodynamic driving force on the reference ion.

Partial core volume and entropy. Equation 60 suffices to define all the properties needed to parameterize the thermodynamic driving forces in equation 64 . The modelling of kinematic phenomena, however, involves volumetric state equations like equation 9 . Thus it is appropriate to consider how variations in excess charge impact parameters such as partial molar volumes.

To get an equation for molar volume from equation 47 , one can take a pressure derivative, following the route by which $V$ was derived from $G$ in equation 2 . Although the composition basis of $\bar{G}$ differs in dimension from that of $G$, Appendix C provides a proof that

$$
\bar{V}_{i}=\left(\frac{\partial \mu_{i}}{\partial p}\right)_{T, n_{i}}=\left(\frac{\partial \mu_{i}}{\partial p}\right)_{T, y_{i<n-1}, \overline{\mathrm{P}}},
$$

that is, one retrieves the same quantity when partially differentiating with $p$, regardless whether composition is held constant by fixing the extensive, $n$-dimensional basis of molar contents $\left\{n_{i}\right\}_{n}$ or the intensive, $(n-1)$-dimensional neutralizable composition basis $\left\{\left\{y_{i}\right\}_{n-2}, \overline{\mathrm{P}}\right\}$.

Hence differentiate Euler equation 47 with respect to pressure at constant temperature and neutralizable composition to produce a volumetric equation of state:

$$
\frac{1}{c_{\mathrm{T}}}=\sum_{i=1}^{n-1}\left(\frac{\partial \mathrm{M}_{i}^{n}}{\partial p}\right)_{T, x_{i}} x_{i}+\left(\frac{\partial \mathrm{M}_{n}^{n}}{\partial p}\right)_{T, x_{i}} .
$$

By similarly differentiating $\left[\mathrm{M}_{i}^{n}\right]_{n}=\mathbf{T}_{n}^{\mathrm{M}^{n} \leftrightarrow \mu}\left[\mu_{i}\right]_{n}$, the matrix equation that defines the transformation constructed in equation 49, one finds after applying equation 67 that

$$
\left[\left(\frac{\partial \mathrm{M}_{i}^{n}}{\partial p}\right)_{T, x_{i}}\right]_{n}=\mathbf{T}_{n}^{\mathrm{M}^{n} \leftarrow \mu}\left[\bar{V}_{i}\right]_{n} .
$$

Each derivative in equation 68 therefore derives in a straightforward way from the set of partial molar volumes.

An analogous argument shows that the set of partial molar entropies can be determined by taking temperature derivatives of the core potentials. Through a set of algebraic manipulations using previous definitions, one finds

$$
\left[\left(\frac{\partial \mathrm{M}_{i}^{n}}{\partial T}\right)_{p, y_{k<n-1}, \overline{\mathrm{P}}}\right]_{n}=-\mathbf{T}_{n}^{\mathrm{M}^{n} \leftrightarrow \mu}\left[\bar{S}_{i}\right]_{n},
$$

where $\bar{S}_{i}$ can be understood in the canonical way - as a partial derivative of $S\left(T, p,\left\{n_{i}\right\}_{n}\right)$ with $n_{i}$ - as well as through the Maxwell relation implied by equation 12 or through an equivalent temperature derivative of $\mu_{i}$ in which the intensive composition basis is held constant.

The definition of some new properties will help to clarify the dimensions of quantities obtained by partially differentiating core potentials. Introduce the symbols

$$
\overline{\mathrm{v}}_{i}^{n}=\left(\frac{\partial \mathrm{M}_{i}^{n}}{\partial p}\right)_{T, x_{i}} \quad \text { and } \quad \overline{\mathrm{s}}_{i}^{n}=-\left(\frac{\partial \mathrm{M}_{i}^{n}}{\partial T}\right)_{p, x_{i}} ;
$$

we call $\left\{\overline{\mathrm{v}}_{i}^{n}\right\}_{n}$ partial core volumes and $\left\{\overline{\mathrm{s}}_{i}^{n}\right\}_{n}$ partial core entropies. Bringing in these new quantities recasts the transformations in equations 69 and 70 as

$$
\left[\overline{\mathrm{v}}_{i}^{n}\right]_{n}=\mathbf{T}_{n}^{\mathrm{M}^{n} \leftrightarrow \mu}\left[\bar{V}_{i}\right]_{n} \quad \text { and } \quad\left[\overline{\mathrm{s}}_{i}^{n}\right]_{n}=\mathbf{T}_{n}^{\mathrm{M}^{n} \leftarrow \mu}\left[\bar{S}_{i}\right]_{n} .
$$

Partial core properties simplify the equations of state for molar volume and molar entropy that arise from core potentials as well. These become

$$
\frac{1}{c_{\mathrm{T}}}=\sum_{i=1}^{n-1} \overline{\mathrm{v}}_{i}^{n} x_{i}+\overline{\mathrm{v}}_{n}^{n} \quad \text { and } \quad \bar{S}=\sum_{i=1}^{n-1} \overline{\mathrm{s}}_{i}^{n} x_{i}+\overline{\mathrm{s}}_{n}^{n}
$$

analogues of equations 9 and 12 which describe, respectively, how molar volume and molar entropy depend on the neutralizable composition basis. To derive the first of these equations one can directly substitute $\left\{\overline{\mathrm{v}}_{i}^{n}\right\}_{n}$ from equation 71 into equation 68 ; the second is obtained by a similar route, which starts by partially differentiating Euler equation 47 with temperature. Alternatively, one can derive both of equations 73 from the Gibbs-Duhem equation. Differentiation of equation 48 by $p$ yields the state equation for $1 / c_{\mathrm{T}}$; differentiation with $T$ yields $-\bar{S}$.

Appendix D provides an analysis of volume and entropy changes, which shows how the partial core volumes and partial core entropies can also be interpreted as thermodynamic derivatives with respect to the neutralizable basis. This reveals a process by which the partial core volumes can be measured experimentally: as well as being given by equation 72 , the subset $\left\{\overline{\mathrm{v}}_{i}^{n}\right\}_{n-1}$ satisfies

$$
\overline{\mathrm{v}}_{i}^{n}=\left(\frac{\partial\left(1 / c_{\mathrm{T}}\right)}{\partial x_{i}}\right)_{T, p, x_{j \neq i, j<n}} .
$$

Thus the charging ion's partial core volume, $\overline{\mathrm{v}}_{n-1}^{n}$, being conjugate to the molar charge, quantifies overall volume change due to piezoelectric effects; the core volumes associated with the chemical state, $\left\{\overline{\mathrm{v}}_{i}^{n}\right\}_{n-2}$, can be established by varying composition at fixed charge. The appendix further shows how to establish $\overline{\mathrm{v}}_{n}^{n}$ given the other partial core volumes, and also discusses partial core entropies.

\section{Thermodynamic constitutive laws}

Core parameters for Guggenheim potentials. With the traditional material parameters redefined to distinguish electrical state effects, it becomes possible to create a constitutive framework for electrochemical-potential changes 
$\left\{d \mu_{i}\right\}_{n}$ wherein electroneutrality can be either adopted or relaxed. As well as producing results consistent with the Guggenheim or Smyrl-Newman thermodynamic formulations for locally electroneutral electrolytic solutions, the new framework allows consistent description of nonneutral situations, such as Gibbs-Donnan equilibria.

Since the particle-fraction and neutralizable bases are connected by an invertible map, $\bar{G}\left(T, p,\left\{x_{i}\right\}_{n-1}\right)$ naturally for an electrolytic fluid. Equation 47 therefore requires that all of the fluid's core potentials have at most the same functional dependence: $\mathrm{M}_{i}^{n}\left(T, p,\left\{x_{i}\right\}_{n-1}\right)$. Consequently, when a fluid electrolyte undergoes a reversible change in state, the change in any core potential can be written as

$$
\begin{aligned}
d \mathrm{M}_{i}^{n}= & \left(\frac{\partial \mathrm{M}_{i}^{n}}{\partial T}\right)_{p, x_{k}} d T+\left(\frac{\partial \mathrm{M}_{i}^{n}}{\partial p}\right)_{T, x_{k}} d p \\
& +\sum_{j=1}^{n-1}\left(\frac{\partial \mathrm{M}_{i}^{n}}{\partial x_{j}}\right)_{T, p, x_{k \neq j}} d x_{j} .
\end{aligned}
$$

Partial core entropies, partial core volumes, and core thermodynamic factors can be introduced to replace the partial derivatives here. The matrix form

$$
d\left[\mathrm{M}_{i}^{n}\right]_{n}=-\left[\overline{\mathrm{s}}_{i}\right]_{n} d T+\left[\overline{\mathrm{v}}_{i}\right]_{n} d p+\overline{\mathbf{X}} d\left[x_{i}\right]_{n-1}
$$

therefore summarizes all the possible ways the core potentials of an electrolytic fluid can be varied reversibly, in terms of parameters measurable over the neutralizable composition basis.

In a similar way, bearing in mind that equations 5 and 8 make it natural to write $\mu_{i}\left(T, p,\left\{y_{i}\right\}_{n-1}\right)$, equation 64 shows that the Guggenheim potentials vary as

$$
d\left[\mu_{i}\right]_{n}=-\left[\bar{S}_{i}\right]_{n} d T+\left[\bar{V}_{i}\right]_{n} d p+\mathbf{y}_{n}^{-1} \mathbf{Q} d\left[y_{i}\right]_{n-1} .
$$

Here $\mathbf{y}_{n}$ is a diagonal $n \times n$ matrix whose $i^{\text {th }}$ diagonal entry is $y_{i}$; the $i^{\text {th }}$ diagonal entry of its inverse, $\mathbf{y}_{n}^{-1}$, is $1 / y_{i}$.

Applying the transformation defined in equation 50 to equation 76 , then inserting equation 61 , gives

$$
\begin{aligned}
d\left[\mu_{i}\right]_{n}= & -\mathbf{T}_{n}^{\mu \leftrightarrow \mathrm{M}^{n}}\left[\overline{\mathrm{s}}_{i}\right]_{n} d T+\mathbf{T}_{n}^{\mu \leftrightarrow \mathrm{M}^{n}}\left[\overline{\mathrm{v}}_{i}\right]_{n} d p \\
& +\mathbf{T}_{n}^{\mu \leftrightarrow \mathrm{M}^{n}} \boldsymbol{\Gamma}_{x} \overline{\mathbf{X}}_{n-1} d\left[x_{i}\right]_{n-1} .
\end{aligned}
$$

This is the second major result of the analysis. It shows how all of the Guggenheim electrochemical potentials can be parameterized in terms of property sets $\left\{\overline{\mathrm{s}}_{i}\right\}_{n},\left\{\overline{\mathrm{v}}_{i}\right\}_{n}$, and the independent entries in symmetric property matrix $\overline{\mathbf{X}}_{n-1}$, each of which is measurable by experiments in which the molar charge is expressly fixed or varied. Furthermore, equation 78 distinguishes the parts of the Guggenheim potentials that vary with electrical state, which are proportional to $d x_{n-1}$ (that is, $d \overline{\mathrm{P}}$ ).

Practical implementations of transport equations can be facilitated by using a basis of $n-1$ diffusion driving forces, which depend on a basis of $n-1$ independent composition gradients. Thus it is useful to state in matrix form a transformation that sends the independent set of Guggenheim potential derivatives $\left\{d \mu_{i}\right\}_{n-1}$ into the independent core potential derivatives $\left\{d \mathrm{M}_{i}^{n}\right\}_{n-1}$. This transformation and its inverse are constructed in Appendix E.
Core parameters for Smyrl-Newman potentials. The SmyrlNewman potentials $\left\{\mu_{i}^{n}\right\}_{n}$ are formed by linearly recombining Guggenheim potentials:

$$
\mu_{i}^{n}=\left\{\begin{array}{rcc}
\mu_{i}-\frac{z_{i}}{z_{n}} \mu_{n} & ; \quad i<n \\
\mu_{n} & ; \quad i=n .
\end{array}\right.
$$

This transformation and its inverse are both linear, expressed in matrix form as

$$
\left[\mu_{i}^{n}\right]_{n}=\mathbf{T}_{n}^{\mu^{n} \leftrightarrow \mu}\left[\mu_{i}\right]_{n} \text { or }\left[\mu_{i}\right]_{n}=\mathbf{T}_{n}^{\mu \leftrightarrow \mu^{n}}\left[\mu_{i}^{n}\right]_{n},
$$

with entries of the transformation matrices given by

$$
\begin{aligned}
& \left(\mathbf{T}_{n}^{\mu^{n} \leftrightarrow \mu}\right)_{i j}=\delta_{i j}-\frac{z_{i}}{z_{n}}\left(1-\delta_{i n}\right) \delta_{n j}, \\
& \left(\mathbf{T}_{n}^{\mu \leftrightarrow \mu^{n}}\right)_{i j}=\delta_{i j}+\frac{z_{i}}{z_{n}}\left(1-\delta_{i n}\right) \delta_{n j} .
\end{aligned}
$$

It follows that the set of differential Smyrl-Newman potentials can be expressed in terms of core properties as

$$
\begin{aligned}
d\left[\mu_{i}^{n}\right]_{n}= & -\mathbf{T}_{n}^{\mu^{n} \leftrightarrow \mathrm{M}^{n}}\left[\overline{\mathrm{s}}_{i}\right]_{n} d T+\mathbf{T}_{n}^{\mu^{n} \leftrightarrow \mathrm{M}^{n}}\left[\overline{\mathrm{v}}_{i}\right]_{n} d p \\
& +\mathbf{T}_{n}^{\mu^{n} \leftrightarrow \mathrm{M}^{n}} \boldsymbol{\Gamma}_{x} \overline{\mathbf{X}}_{n-1} d\left[x_{i}\right]_{n-1} .
\end{aligned}
$$

This is almost identical to equation 78 , except that the transformation matrix

$$
\begin{aligned}
\left(\mathbf{T}_{n}^{\mu^{n} \leftrightarrow \mathrm{M}^{n}}\right)_{i j}= & \left(\mathbf{T}_{n}^{\mu^{n} \leftrightarrow \mu} \mathbf{T}_{n}^{\mu \leftrightarrow \mathrm{M}^{n}}\right)_{i j} \\
= & \delta_{i j}\left(1-\delta_{(n-1) j}\right)+\frac{z_{i} \delta_{i n} \delta_{(n-1) j}}{z_{n}-z_{n-1}} \\
& +\frac{z_{n}-z_{i}}{z_{n}}\left(1-\delta_{i n}\right) \delta_{n j}
\end{aligned}
$$

has different entries.

Smyrl and Newman intend the potentials $\left\{\mu_{i}^{n}\right\}_{n-1}$ to be independent of the electrical state [20], but this only holds true under special circumstances. Consider the SmyrlNewman potential of the charging ion; a little algebraic rearrangement involving the symmetry of the core thermodynamic factors shows that it varies as

$$
\begin{aligned}
-\frac{z_{n} d \mu_{n-1}^{n}}{z_{n}-z_{n-1}}= & \sum_{k=1}^{n-2} \bar{X}_{(n-1) k} d\left(x_{k} x_{n-1}\right)+ \\
& \frac{1}{2} \bar{X}_{(n-1)(n-1)} d\left(x_{n-1}\right)^{2}+ \\
& \sum_{k, l=1}^{n-2} x_{k} \bar{X}_{k l} d x_{l} .
\end{aligned}
$$

Under the electroneutrality approximation $\left(x_{n-1}=0\right)$, the first two terms on the right of the equals sign vanish, leaving a functionality consistent with Smyrl and Newman's claim. But if electroneutrality is relaxed, the SmyrlNewman chemical potential of the charging ion can only be independent of the electrical state if $\bar{X}_{(n-1) j}=0$ for all $j$, that is, if the naive electric potential is independent of both composition and molar excess charge. 


\section{Electrical colligative properties}

Importantly, all the entries in the symmetric submatrix obtained by deleting the $(n-1)^{\text {st }}$ row and column of $\overline{\mathbf{X}}_{n-1}$ can be established unambiguously by thermodynamic measurements at constant charge density. Practically speaking, this means that the truncated matrix, $\overline{\mathbf{X}}_{n-2}$, can be established by performing measurements on electroneutral systems. Thus the thermodynamic data currently used to describe concentrated electrolytes appear explicitly in the constitutive framework.

Some uncertainty remains about how to define the properties that appear in the last row and column of $\overline{\mathbf{X}}_{n-1}$, which involve derivatives of the naive electric potential with composition, or derivatives of chemical potentials with respect to charge. Of particular concern is the property

$$
\overline{\mathrm{X}}_{(n-1)(n-1)}=F^{2}\left(z_{n}-z_{n-1}\right)^{2}\left(\frac{\partial \Phi}{\partial \overline{\mathrm{P}}}\right)_{T, p, y_{k<n-1}}
$$

that appears in equation 84 . As mentioned just after that equation, one could ensure the consistency of Smyrl and Newman's formalism by insisting that $\overline{\mathrm{P}}$ and $\Phi$ are independent. But many well-established theories, including the Gouy-Chapman analysis of diffuse double layers, the Debye-Hückel model of electrolyte activity, and the DLVO treatment of disjoining force, begin by asserting a state equation for $\overline{\mathrm{P}}(\Phi)$. If a nontrivial relationship of this sort exists, then the property in equation 85 cannot vanish, and must be specified in a thermodynamically consistent way.

Below we discuss two equations of state for molar excess charge and corresponding forms for the electrical core thermodynamic factors. First, the ideal-solution approximation for species activities is shown to induce an equation of state that relates charge to the naive potential. Second, the equation of state for charge used in typical PoissonBoltzmann theory is shown to induce equations of state for the electrical core thermodynamic factors. Both of these state equations are shown to exhibit behavior that contradicts typical thermodynamic understanding in some regimes. It should be emphasized that many other state equations can be used, but these examples suffice to show the main routes by which electrical properties can be specified within the present framework.

The neutral corresponding state. Before proceeding with the illustrative examples, we begin with a more general discussion of how the choice of a two-ion secondary reference state can be used to establish natural reference points for overall composition and naive potential. Once a reference ion/charging ion pair is specified, these reference points can be selected in an unambiguous way, without requiring the adoption of a particular theory to underpin the electrical colligative properties.

Whenever $y_{n} \neq 0$, to any chemical composition $\left\{y_{i}\right\}_{n-2}$ there corresponds a unique set of $n$ particle fractions, written $\left\{y_{i}^{0}\right\}_{n}$, for which $x_{n-1}=0$. Using equations 5 and 25 , this electroneutral reference composition in the two-ion secondary reference state can be defined explicitly as

$$
y_{i}^{0}=\left\{\begin{array}{cl}
y_{i} & ; \quad i<n-1 \\
\frac{z_{n}}{z_{n}-z_{n-1}}-\sum_{k=1}^{n-2}\left(\frac{z_{n}-z_{k}}{z_{n}-z_{n-1}}\right) y_{k} & ; \quad i=n-1 \\
-\frac{z_{n-1}}{z_{n}-z_{n-1}}-\sum_{k=1}^{n-2}\left(\frac{z_{k}-z_{n-1}}{z_{n}-z_{n-1}}\right) y_{k} & ; \quad i=n .
\end{array}\right.
$$

The uniqueness of this corresponding state ensures that choosing a reference ion and charging ion also specifies a particular electroneutral reference composition that corresponds to any overall composition - whether that composition be neutral or non-neutral.

The process of deriving state equations or thermodynamic factors is facilitated by introducing the electroneutral reference composition. In particular, it follows from the choice of a two-ion secondary reference state that the two particle fractions

$$
y_{n-1}=y_{n-1}^{0}-x_{n-1} \text { and } y_{n}=y_{n}^{0}+x_{n-1}
$$

depend on the molar excess charge, while every other particle fraction does not.

There must always exist some value of the naive potential that corresponds to the electroneutral reference composition. We notate this as $\Phi_{0}$ and call it the electroneutral potential established by the two-ion secondary reference state. When analyzing state equations for charge, the naive potential can be partitioned as

$$
\Phi\left(\left\{x_{i}\right\}_{n-1}\right)=\Phi_{0}\left(\left\{x_{i}\right\}_{n-2}\right)-\Phi_{\mathrm{P}}\left(\left\{x_{i}\right\}_{n-1}\right),
$$

in which $\Phi_{\mathrm{P}}$ is the Poisson overpotential, i.e. the potential that contributes to electrical non-neutrality. This is negative because non-neutrality is typically thought of as being imposed by an externally applied voltage, whereas the naive potential and electroneutral potential measure internal electrical energy. This distinction becomes important later, since equations of state for excess charge generally show that charge decreases with (external) potential, whereas the positive semidefinite property of $\overline{\mathbf{K}}_{n-1}$ suggests that $\bar{X}_{(n-1)(n-1)}$ should not be negative.

In the following analysis it will be helpful to employ the concept of ionic strength. Rather than using the quantity originally defined by Lewis and Randall [56], which assumes that ion concentrations are Boltzmann distributed with respect to Poisson overpotential, we define the neutralizable molar ionic strength with respect to the two-ion reference state, $\bar{I}$, through a thermodynamic derivative,

$$
\bar{I}=-\frac{R T}{2 F^{2}}\left(\frac{\partial \overline{\mathrm{P}}}{\partial \Phi_{\mathrm{P}}}\right)_{T, p, y_{k<n-1}} .
$$

The neutral molar ionic strength $\bar{I}_{0}$ obtains when this derivative is evaluated at $\Phi_{\mathrm{P}}=0$. Although more abstract, equation 89 provides a general definition commensurate with the use of the ionic-strength concept by Lewis and Randall, Debye and Hückel, Guggenheim, Pitzer, and others, but which does not require one to assume that a particular state equation governs the charge density. 
Ideal naive potential. One way to develop non-neutral thermodynamic constitutive equations is to assume facts about species' activities, then use these to deduce an equation of state for charge. This first example shows how specifying the composition dependence of species' excess free energies of mixing fixes the electrical terms in $\overline{\mathbf{X}}_{n-1}$, which further mandates how charge depends on electric potential.

In non-electrochemical thermodynamics, chemical potentials are typically assumed to follow constitutive laws of the form

$$
\mu_{i}=\mu_{i}^{\theta}+R T \ln a_{i}=\mu_{i}^{\theta}(T, p)+R T \ln \gamma_{i} y_{i},
$$

in which $R$ is the gas constant; $\mu_{i}^{\theta}$ is the chemical potential in a standard reference state, and $a_{i}$ is the activity of species $i$; parameters $\gamma_{i}\left(T, p,\left\{y_{i}\right\}_{n-1}\right)$ are activity coefficients on a mole-fraction basis. Partial differentiation of this law for species $i<n$ with respect to the composition of species $j<n$ shows that

$$
\left(\overline{\mathbf{Q}}_{n-1}\right)_{i j}=R T\left[\delta_{i j}+y_{i}\left(\frac{\partial \ln \gamma_{i}}{\partial y_{j}}\right)_{T, p, y_{k \neq j, n}}\right] .
$$

By bringing in activity coefficients, this clarifies the earlier assertion that $\overline{\mathbf{Q}}_{n-1}$ is a multicomponent extension of the binary thermodynamic factor created by Darken [49].

Within ideal-gas mixtures and ideal liquid solutions, the excess free energy of mixing for species $i$ goes as $\ln y_{i}$. Thus the activity coefficients of all species are constant with respect to composition and $\overline{\mathbf{Q}}_{n-1}=R T \mathbf{I}_{n-1}$. Applying this assumption within equation 60 produces

$$
\overline{\mathbf{X}}_{n-1}^{\mathrm{I}}=R T\left(\mathbf{T}_{n-1}^{d x \leftrightarrow d y}\right)^{\mathrm{T}} \mathbf{Y}_{n-1} \mathbf{T}_{n-1}^{d x \leftrightarrow d y}
$$

where the superscript I indicates a property derived under the ideal-solution approximation. Terms in the $(n-1)^{\mathrm{st}}$ row of this equation are given by

$$
\begin{aligned}
\bar{X}_{(n-1) j}^{\mathrm{I}}= & \left(\frac{z_{n}-z_{j}}{z_{n}-z_{n-1}}\right) \frac{R T}{y_{n-1}} \\
& -\left(\frac{z_{j}-z_{n-1}}{z_{n}-z_{n-1}}-\delta_{(n-1) j}\right) \frac{R T}{y_{n}} .
\end{aligned}
$$

One can use equations 5 and 25 to show that this relationship follows if the function

$$
\Phi^{\mathrm{I}}=\frac{R T}{F\left(z_{n}-z_{n-1}\right)} \ln \left(\frac{y_{n}}{y_{n-1}}\right)
$$

defines the ideal naive potential.

After bringing in the electroneutral reference composition, inversion of equation 94 produces a state equation for molar charge. Substitute the particle fractions from equation 87 into equation 94 , then isolate $x_{n-1}$ to find

$$
\begin{aligned}
x_{n-1}= & \frac{y_{n-1}^{0}}{2}\left\{1+\tanh \left[\frac{F\left(z_{n}-z_{n-1}\right) \Phi^{\mathrm{I}}}{2 R T}\right]\right\} \\
& -\frac{y_{n}^{0}}{2}\left\{1-\tanh \left[\frac{F\left(z_{n}-z_{n-1}\right) \Phi^{\mathrm{I}}}{2 R T}\right]\right\},
\end{aligned}
$$

the desired equation of state.

By considering the electroneutral condition, equation 95 can be solved for the electroneutral potential. Partition $\Phi^{\mathrm{I}}$ in equation 95 according to equation 88 , then note that both charge and the Poisson overpotential vanish in the electroneutral state. After some rearrangement,

$$
\Phi_{0}^{\mathrm{I}}=\frac{R T}{F\left(z_{n}-z_{n-1}\right)} \ln \left(\frac{y_{n}^{0}}{y_{n-1}^{0}}\right)
$$

proves to determine the ideal electroneutral potential.

Therefore the naive potential in an ideal electrolytic fluid is written in terms of the chemical composition as

$$
\Phi^{\mathrm{I}}=\frac{R T}{F\left(z_{n}-z_{n-1}\right)} \ln \left(\frac{y_{n}^{0}}{y_{n-1}^{0}}\right)-\Phi_{\mathrm{P}}^{\mathrm{I}},
$$

where $\Phi_{\mathrm{P}}^{\mathrm{I}}$ is defined through the partitioning in equation 88. Inserting this back into equation 95 shows that

$$
\overline{\mathrm{P}}=-\frac{2 F\left(z_{n}-z_{n-1}\right) y_{n-1}^{0} y_{n}^{0} \tanh \left(\frac{F\left(z_{n}-z_{n-1}\right) \Phi_{\mathrm{P}}^{\mathrm{I}}}{2 R T}\right)}{y_{n-1}^{0}+y_{n}^{0}+\left(y_{n-1}^{0}-y_{n}^{0}\right) \tanh \left(\frac{F\left(z_{n}-z_{n-1}\right) \Phi_{\mathrm{P}}^{\mathrm{I}}}{2 R T}\right)},
$$

an equation of state that specifies how the ideal electrolytic fluid's molar excess charge varies with respect to the Poisson overpotential.

Although the assumption that $\overline{\mathbf{Q}}_{n-1}=R T \mathbf{I}_{n-1}$ closes out the parameterization of $\overline{\mathbf{X}}_{n-1}$ in a thermodynamically consistent way through equation 93 , equations 96 through 98 are somewhat jarring, because they disagree with assumptions that underlie well-accepted Poisson-Boltzmann theories. Particularly troubling is the ideal electrolytic solution's neutral molar ionic strength,

$$
\bar{I}_{0}^{\mathrm{I}}=\frac{\left(z_{n}-z_{n-1}\right)^{2} y_{n-1}^{0} y_{n}^{0}}{2\left(y_{n-1}^{0}+y_{n}^{0}\right)},
$$

which depends only on the electroneutral fractions of the reference ion and charging ion, and appears to involve a harmonic mean of particle fractions, rather than a chargeweighted arithmetic mean. These features disagree with widely adopted and experimentally supported theories, including the activity models of Guggenheim [57] and Pitzer [58], both of which fit colligative properties of multi-salt solutions well using a Lewis-Randall ionic strength that includes all ions.

Poisson-Boltzmann thermodynamic factors. An alternative way to develop non-neutral thermodynamic constitutive equations is to assume an equation of state for charge to be given, then use this to deduce facts about species' activities. To retrieve constitutive laws consistent with Poisson-Boltzmann theories, begin by assuming that the electroneutral state is the reference point for naive potential, $\Phi_{0}^{\mathrm{B}}=0$. Further assume

$$
\overline{\mathrm{P}}=F \sum_{k=1}^{n} z_{k} y_{k}^{0} \exp \left(-\frac{F z_{k} \Phi_{\mathrm{P}}^{\mathrm{B}}}{R T}\right),
$$


that is, that all the species particle fractions relative to the electroneutral reference composition are Boltzmann distributed with respect to the Poisson overpotential. Here the superscript B indicates a property derived under this assumed Boltzmann statistics.

At a given electroneutral composition, charge equation of state 100 is monotonic with respect to $\Phi_{\mathrm{P}}^{\mathrm{B}}$ - the partial derivative $\partial \overline{\mathrm{P}} / \partial \Phi_{\mathrm{P}}^{\mathrm{B}}$ is always negative. Together, the monotonicity of $\overline{\mathrm{P}}$ and its behavior in the limits as $\Phi_{\mathrm{P}}^{\mathrm{B}} \rightarrow \pm \infty$ imply that the relationship between $\Phi_{\mathrm{P}}^{\mathrm{B}}$ and $\overline{\mathrm{P}}$ at a given chemical composition is both one-to-one and onto. Consequently one can speak unambiguously of an inverse function $\Phi_{\mathrm{P}}^{\mathrm{B}}\left(\left\{y_{i}\right\}_{n-2}, \overline{\mathrm{P}}\right)$, despite not being able to write this inverse easily in a closed form. Equation 89 gives the neutralizable ionic strength as

$$
\bar{I}^{\mathrm{B}}=\frac{1}{2} \sum_{k=1}^{n} z_{k}^{2} y_{k}^{0} \exp \left(-\frac{F z_{k} \Phi_{\mathrm{P}}^{\mathrm{B}}}{R T}\right) .
$$

This matches canonical definitions when $\Phi_{\mathrm{P}}^{\mathrm{B}}=0$; for example, at null Poisson overpotential one can recognize the ionic strength Newman defines as $I^{\prime}[22]$ to be $I^{\prime}=c_{\mathrm{T}} \bar{I}_{0}^{\mathrm{B}}$.

Since $\Phi_{0}^{\mathrm{B}}=0$ by design, the overpotential in equation 100 relates simply to the naive potential, through

$$
\Phi^{\mathrm{B}}=-\Phi_{\mathrm{P}}^{\mathrm{B}} .
$$

One can determine the electrical thermodynamic factors $\bar{X}_{(n-1) j}^{\mathrm{B}}$ uniquely by implicit differentiation of $\overline{\mathrm{P}}$ with the neutralizable composition basis variables. Bearing in mind that $\mathrm{M}_{n-1}^{n}=F\left(z_{n}-z_{n-1}\right) \Phi^{\mathrm{B}}=-F\left(z_{n}-z_{n-1}\right) \Phi_{\mathrm{P}}^{\mathrm{B}}$ and $\overline{\mathrm{P}}=$ $F\left(z_{n}-z_{n-1}\right) x_{n-1}$, one finds by partially differentiating equation 100 with respect to $x_{j}$ that

$$
\begin{aligned}
\bar{X}_{(n-1) j}^{\mathrm{B}}= & \frac{R T}{2 \bar{I}^{\mathrm{B}}}\left[\left(z_{n}-z_{n-1}\right)^{2} \delta_{(n-1) j}+\right. \\
& z_{j} z_{n-1}\left(e^{-z_{j} \phi^{\mathrm{B}}}-e^{-z_{n-1} \phi^{\mathrm{B}}}\right)+ \\
& z_{n-1} z_{n}\left(e^{-z_{n-1} \phi^{\mathrm{B}}}-e^{-z_{n} \phi^{\mathrm{B}}}\right)+ \\
& \left.z_{n} z_{j}\left(e^{-z_{n} \phi^{\mathrm{B}}}-e^{-z_{j} \phi^{\mathrm{B}}}\right)\right],
\end{aligned}
$$

in which the variable $\phi$, defined for convenience as

$$
\phi=\frac{F \Phi_{\mathrm{P}}}{R T},
$$

represents a dimensionless Poisson overpotential.

When considered near an electroneutral state, the definition in equation 103 has some appealing features. The neutralizable ionic strength is independent of the chosen reference-ion/charging-ion pair, and all the electrical thermodynamic factors scale inversely with neutralizable ionic strength. Also, when $\overline{\mathrm{P}}=\Phi_{\mathrm{P}}^{\mathrm{B}}=0$, the thermodynamicfactor matrix $\overline{\mathbf{X}}_{n-1}^{\mathrm{B}}$ becomes block diagonal, with a positive entry in the $(n-1)(n-1)$ position being the only nonzero entry in that row and column.
Definition 103 is not a panacea, however. The PoissonBoltzmann neutralizable ionic strength, $\bar{I}^{\mathrm{B}}$, increases exponentially when the Poisson overpotential has sufficiently large magnitude. Therefore the off-diagonal thermodynamic factors $\bar{X}_{(n-1) j}^{\mathrm{B}}$ with $j<n-1$ can approach constant, nonzero values in the high-potential limit, whereas the $\bar{X}_{(n-1)(n-1)}^{\mathrm{B}}$ entry always falls ever closer toward zero. Thus the thermodynamic-factor matrix $\overline{\mathbf{X}}_{n-1}^{\mathrm{B}}$ which derives from the Poisson-Boltzmann equation of state is not necessarily positive definite. In many cases - including the important example of a simple binary electrolytic solution of a 1:1 salt in a neutral solvent — one finds a range of Poisson overpotential in which the thermodynamic factors in equation 103 require $\overline{\mathbf{X}}_{n-1}^{\mathrm{B}}$ to have a negative determinant. This in turn implies that the thermodynamicfactor matrix must afford at least one negative eigenvalue; through the congruence in equation 59, it follows that there must be a potential range where the molar Gibbs function is nonconvex over the particle fractions.

\section{The nonideal $z: z$ Poisson-Boltzmann electrolyte}

To make the abstract analysis given up to this point concrete, it is useful to flesh out the discussion with an example. In this section we therefore develop electrochemicalpotential constitutive laws for a binary electrolytic solution of a symmetric simple salt in a neutral solvent. These thermodynamically consistent forms of the electrochemical potentials will account for chemical nonideality in the electrically neutral state, and will also be consistent with the Poisson-Boltzmann charge/potential relation, equation 100, should electroneutrality be violated.

In this discussion we assume an isothermal, isobaric situation for simplicity. The electrolytic solution in question comprises three species - solvent, cations, and anions - which we designate with symbols $0,+$, and - , respectively. Since the solvent is electroneutral, $z_{0}=0$; since the simple salt is symmetric, the equivalent charges of its constituent ions satisfy $z_{+}=-z_{-}=z$. We establish the two-ion secondary reference state by adopting a species ordering $\{0,+,-\}$, which indicates that the anion $(-)$ is the reference ion and the cation $(+)$ is the charging ion.

Particle fractions of all the species in the electroneutral reference state are established through equation 86 , which shows that

$$
y_{i}^{0}=\left\{\begin{array}{cll}
y_{0} & ; \quad i=0 \\
\frac{1}{2}\left(1-y_{0}\right) & ; \quad i=+,-
\end{array}\right.
$$

With this in hand, it is possible to rewrite equation 100 for the example as

$$
\frac{\overline{\mathrm{P}}}{F z}=-\left(1-y_{0}\right) \sinh (z \phi),
$$

in which the dimensionless Poisson overpotential $\phi$ is defined by equation 104 . 
The purely electrical colligative properties can be determined by leveraging equations 105 and 106. Application of equation 89 shows that the ionic strength in this case is given by

$$
\bar{I}^{\mathrm{B}}=\frac{1}{2} z^{2}\left(1-y_{0}\right) \cosh (z \phi) .
$$

Equation 103 can then be analyzed by inserting the relationship for $\bar{I}^{\mathrm{B}}$, then taking $j=0$ and $j=+$. This process shows that the entries of Hessian $\overline{\mathbf{X}}_{+}$in the column corresponding to the charging ion are

$$
\bar{X}_{+0}^{\mathrm{B}}=\frac{2 R T \tanh (z \phi)}{1-y_{0}} \text { and } \bar{X}_{++}^{\mathrm{B}}=\frac{4 R T \operatorname{sech}(z \phi)}{1-y_{0}} .
$$

The symmetry shown in equation 57 fixes another entry of the $2 \times 2$ matrix $\overline{\mathbf{X}}_{+}$, since $\bar{X}_{0+}^{\mathrm{B}}=\bar{X}_{+0}^{\mathrm{B}}$. Thus, specifying that the electrolyte in question satisfies the PoissonBoltzmann charge/potential relation fixes three of the four entries in $\overline{\mathbf{X}}_{+}$explicitly. Only the single free parameter $\bar{X}_{00}^{\mathrm{B}}$ remains.

A simple parameterization for differentials of the electrochemical potentials is produced as follows. Start with the isothermal, isobaric version of equation 17 , then inject transformation 26 to move to the neutralizable composition basis:

$$
\begin{aligned}
{\left[\begin{array}{l}
y_{0} d \mu_{0} \\
y_{+} d \mu_{+}
\end{array}\right] } & =\overline{\mathbf{Q}}_{+} d\left[\begin{array}{c}
y_{0} \\
y_{+}
\end{array}\right] \\
& =\overline{\mathbf{Q}}_{+} \mathbf{T}_{+}^{d y \leftrightarrow d x} d\left[\begin{array}{c}
y_{0} \\
-\frac{1}{2 F z} \overline{\mathrm{P}}
\end{array}\right] .
\end{aligned}
$$

Next, using equation 60, replace the Darken matrix $\overline{\mathbf{Q}}_{+}$ with the Hessian $\overline{\mathbf{X}}_{+}$, all but one of whose entries is known. Then perform a Gibbs-Duhem extension by multiplying through with $\boldsymbol{\Gamma}_{1}$ from equation 66 to get

$$
\left[\begin{array}{l}
y_{0} d \mu_{0} \\
y_{+} d \mu_{+} \\
y_{-} d \mu_{-}
\end{array}\right]=\boldsymbol{\Gamma}_{1} \mathbf{Y}_{+}^{-1}\left(\mathbf{T}_{+}^{d y \leftrightarrow d x}\right)^{\mathrm{T}} \overline{\mathbf{X}}_{+} d\left[\begin{array}{c}
y_{0} \\
-\frac{1}{2 F z} \overline{\mathrm{P}}
\end{array}\right] \text {. }
$$

Here, the product of three matrices $\boldsymbol{\Gamma}_{1} \mathbf{Y}_{+}^{-1}\left(\mathbf{T}_{+}^{d y \leftrightarrow d x}\right)^{\mathrm{T}}$ can be worked out by exploiting the indicial forms from equations 19,27 , and 66 , ultimately showing that

$$
\begin{aligned}
& \boldsymbol{\Gamma}_{1} \mathbf{Y}_{+}^{-1}\left(\mathbf{T}_{+}^{d y \leftrightarrow d x}\right)^{\mathrm{T}}= \\
& {\left[\begin{array}{cc}
y_{0}\left(1-y_{0}\right) & y_{0}\left[y_{+}-\frac{1}{2}\left(1-y_{0}\right)\right] \\
-y_{+} y_{0} & y_{+}\left[\frac{1}{2} y_{0}-\left(1-y_{+}\right)\right] \\
-\left(1-y_{0}-y_{+}\right) y_{0} & \left(1-y_{0}-y_{+}\right)\left(\frac{1}{2} y_{0}+y_{+}\right)
\end{array}\right] .}
\end{aligned}
$$

Although this expression involves the particle-fraction basis $\left\{y_{0}, y_{+}\right\}$, it can be recast in terms of the neutralizable basis by using equations 5 and 23 to produce

$$
\begin{aligned}
y_{+} & =\frac{1}{2}\left(\frac{1}{F z} \overline{\mathrm{P}}\right)+\frac{1}{2}\left(1-y_{0}\right) \\
& =\frac{1}{2}\left(1-y_{0}\right)[1-\sinh (z \phi)] .
\end{aligned}
$$

The latter equality here exploits equation 106 to interchange charge density and Poisson overpotential.
Equation 110 allows one to determine the standard electroneutral thermodynamic factors used by Newman in his concentrated solution theory [22]. Setting the excess charge equal to zero in equation 110 produces

$$
\left.\left[\begin{array}{l}
y_{0} d \mu_{0} \\
y_{+} d \mu_{+} \\
y_{-} d \mu_{-}
\end{array}\right]\right|_{\overline{\mathrm{P}}=0}=\left.\left[\boldsymbol{\Gamma}_{1} \mathbf{Y}_{+}^{-1}\left(\mathbf{T}_{+}^{d y \leftrightarrow d x}\right)^{\mathrm{T}} \overline{\mathbf{X}}_{+}\right]\right|_{\overline{\mathrm{P}}=0} d\left[\begin{array}{c}
y_{0}^{0} \\
0
\end{array}\right]
$$

Since the last entry of the neutralizable composition vector on the right vanishes, the last column of the product of matrices on the right does not contribute here. The matrix containing the nontrivial coefficients is identified as the Newman matrix $\chi_{+}$, through the defining equation

$$
\left.\left[\boldsymbol{\Gamma}_{1} \mathbf{Y}_{+}^{-1}\left(\mathbf{T}_{+}^{d y \leftrightarrow d x}\right)^{\mathrm{T}} \overline{\mathbf{X}}_{+}\right]\right|_{\overline{\mathrm{P}}=0} d\left[\begin{array}{c}
y_{0}^{0} \\
0
\end{array}\right]=R T \boldsymbol{\chi}_{+} d\left[\begin{array}{l}
y_{0}^{0}
\end{array}\right]
$$

Observe that Newman's electroneutral thermodynamic factors are generally quantified by an $n \times(n-2)$ matrix; in this case, $\chi_{+}$is $3 \times 1$.

Insertion of the Poisson-Boltzmann colligative properties from equation 108 and the neutral composition from equation 105 shows that

$$
\chi_{+}=\left[\begin{array}{c}
\chi_{00} \\
\chi_{+0} \\
\chi_{-0}
\end{array}\right]=\left.\frac{1}{R T}\left[\begin{array}{c}
y_{0}\left(1-y_{0}\right) \bar{X}_{00}^{\mathrm{B}} \\
-\frac{1}{2} y_{0}\left(1-y_{0}\right) \bar{X}_{00}^{\mathrm{B}} \\
-\frac{1}{2} y_{0}\left(1-y_{0}\right) \bar{X}_{00}^{\mathrm{B}}
\end{array}\right]\right|_{\overline{\mathrm{P}}=0} .
$$

Thus all $3(3-1) / 2=3$ electroneutral thermodynamic factors depend on just $(3-1)(3-2) / 2=1$ electroneutral core thermodynamic factor, because only the material property $\left.\bar{X}_{00}^{\mathrm{B}}\right|_{\mathrm{P}=0}$ remains after discarding quantities associated with the electrical state from equation 113.

Equation 115 shows that $\chi_{00}=-2 \chi_{+0}=-2 \chi_{+0}=\chi_{0}$, where $\chi_{0}$ parametrically represents all three factors in the electroneutral state. Inspection of equation 113 reveals that the property $\chi_{0}$ so defined also identifies with the thermodynamic factor typically used by Newman for binary electrolytes [22], as expressed over the particle-fraction composition basis by Liu and Monroe [59].

To emphasize how existing thermodynamic correlations can be incorporated we state two definitions. First, introduce a non-neutral Newman thermodynamic factor $\chi$, which generally depends on both the chemical composition and charge,

$$
\chi\left(y_{0}, \overline{\mathrm{P}}\right)=\frac{y_{0}\left(1-y_{0}\right) \bar{X}_{00}^{\mathrm{B}}}{R T} .
$$

Second, partition this factor so that it matches $\chi_{0}$ at the electroneutral reference composition: let

$$
\chi=\chi_{0}\left(y_{0}^{0}\left(y_{0}, \overline{\mathrm{P}}\right)\right)+\chi_{\mathrm{e}}\left(y_{0}, \overline{\mathrm{P}}\right), \text { where } \chi_{\mathrm{e}}\left(y_{0}, 0\right)=0 \text {. }
$$

These definitions allow the single free core thermodynamic factor $\bar{X}_{00}^{\mathrm{B}}$ to be replaced with a simpler parameter $\chi$, 
while also delineating the portion of its behaviour that is known from traditional thermodynamic measurements.

Tables showing how experimental mean molar or mean molal activity coefficients vary with composition are widely available $[1,22,54,58,60]$. When densitometric data are also to hand, such tables can be fit to produce $\chi_{0}$ as a function of $y_{0}^{0}$. The extensive tables of Chapman and Newman [60], for example, provide enough information to determine $\chi_{0}$ functions for many aqueous electrolytes. As a more concrete illustration, Hou and Monroe [61] and Wang et al. [62] recently studied $\chi_{0}$ using a suite of techniques that includes densitometry, Hittorf experiments, and the measurement of steady liquid-junction potentials in concentration cells, finding that the correlation

$$
\begin{aligned}
\chi_{0}= & 1-1.877\left(1-y_{0}^{0}\right)^{1 / 2} \\
& +13.12\left(1-y_{0}^{0}\right)+131.7\left(1-y_{0}^{0}\right)^{7 / 2}
\end{aligned}
$$

quantifies the chemical nonideality of electroneutral solutions of $\mathrm{LiPF}_{6}$ in propylene carbonate. This expression is valid from $y_{0}^{0}=1$ (infinite dilution) down to $y_{0}^{0}=0.64$ (a salt concentration of $\sim 2 \mathrm{M}$ ). Hou and Monroe's methodology applies to different salt/solvent systems as well: Wang et al. [62] provide a similar correlation for $\mathrm{LiPF}_{6}$ in ethylmethyl carbonate. Neutral thermodynamic factors for various other nonaqueous lithium-ion electrolytes have been measured as functions of salt molarity and temperature by Landesfeind et al. [63]; with appropriate densitometric information those data could also be used to determine $\chi_{0}\left(y_{0}^{0}\right)$.

Next we return to the generally non-neutral situation. Use equation 112 to eliminate $\phi$ in favor of $\overline{\mathrm{P}}$ in equation 108 , and $y_{+}$in favor of $\overline{\mathrm{P}}$ in equation 111 . Then insert equations 108, 111, 116, and 117 into equation 110 to get

$$
\begin{aligned}
d \mu_{0}= & R T\left[\chi_{0}+\chi_{\mathrm{e}}-\frac{y_{0}\left(\frac{1}{F z} \overline{\mathrm{P}}\right)^{2}}{\left(1-y_{0}\right) \sqrt{\left(1-y_{0}\right)^{2}+\left(\frac{1}{F z} \overline{\mathrm{P}}\right)^{2}}}\right] d \ln y_{0}, \\
d \mu_{+}= & R T\left[\chi_{0}+\chi_{\mathrm{e}}+\frac{\left(\frac{1}{F z} \overline{\mathrm{P}}\right)^{2}-\left(\frac{1}{F z} \overline{\mathrm{P}}\right)}{\sqrt{\left(1-y_{0}\right)^{2}+\left(\frac{1}{F z} \overline{\mathrm{P}}\right)^{2}}}\right] d \ln \left(1-y_{0}\right) \\
& +\frac{R T\left[\left(1-y_{0}\right)-\left(\frac{1}{F z} \overline{\mathrm{P}}\right)\right]}{\left(1-y_{0}\right) \sqrt{\left(1-y_{0}\right)^{2}+\left(\frac{1}{F z} \overline{\mathrm{P}}\right)^{2}}} d\left(\frac{1}{F z} \overline{\mathrm{P}}\right), \\
d \mu_{-}= & R T\left\{\chi_{0}+\chi_{\mathrm{e}}+\frac{\left(\frac{1}{F z} \overline{\mathrm{P}}\right)^{2}+\left(\frac{1}{F z} \overline{\mathrm{P}}\right)}{\left.\sqrt{\left(1-y_{0}\right)^{2}+\left(\frac{1}{F z} \overline{\mathrm{P}}\right)^{2}}\right\}}\right\} \ln \left(1-y_{0}\right) \\
& -\frac{R T\left[\left(1-y_{0}\right)+\left(\frac{1}{F z} \overline{\mathrm{P}}\right)\right]}{\left(1-y_{0}\right) \sqrt{\left(1-y_{0}\right)^{2}+\left(\frac{1}{F z} \overline{\mathrm{P}}\right)^{2}}} d\left(\frac{1}{F z} \overline{\mathrm{P}}\right) .
\end{aligned}
$$

Observe that charge density appears in all three expressions - even the one that describes the neutral solvent.

A more familiar set of equations can be derived by casting equations 119 in terms of electric potential, rather than charge. Use equation 106 to eliminate excess molar charge in favor of Poisson overpotential to reveal that

$$
\begin{aligned}
d \mu_{0}= & R T\left[\chi_{0}+\chi_{\mathrm{e}}-\frac{y_{0} \sinh ^{2}(z \phi)}{\cosh (z \phi)}\right] d \ln y_{0}, \\
d \mu_{+}= & R T\left[\chi_{0}+\chi_{\mathrm{e}}-\frac{y_{0} \sinh ^{2}(z \phi)}{\cosh (z \phi)}\right] d \ln \left(1-y_{0}\right) \\
& -R T z[1+\sinh (z \phi)] d \phi, \\
d \mu_{-}= & R T\left[\chi_{0}+\chi_{\mathrm{e}}-\frac{y_{0} \sinh ^{2}(z \phi)}{\cosh (z \phi)}\right] d \ln \left(1-y_{0}\right) \\
& +R T z[1-\sinh (z \phi)] d \phi .
\end{aligned}
$$

The first of these expressions contains no $d \phi$ term, which implies that the solvent chemical potential must be independent of the Poisson overpotential, as we might expect for any neutral species.

For the neutral-species chemical potential to be independent of charge state at any chemical composition, one must have that the partial derivative $\partial \mu_{0} / \partial \ln y_{0}$ is independent of $\phi$. To meet this requirement, choose the functionality of $\chi_{\mathrm{e}}\left(y_{0}, \overline{\mathrm{P}}\right)$ such that

$$
\chi_{\mathrm{e}}=\frac{y_{0} \sinh ^{2}(z \phi)}{\cosh (z \phi)}=\frac{y_{0}\left(\frac{1}{F z} \overline{\mathrm{P}}\right)^{2}}{\left(1-y_{0}\right) \sqrt{\left(1-y_{0}\right)^{2}+\left(\frac{1}{F z} \overline{\mathrm{P}}\right)^{2}}}
$$

This choice has the ancillary benefit of addressing the concern raised earlier, that the Poisson-Boltzmann equation of state may force the Gibbs function to be nonconvex. By including equation 121 , one finds that the Hessian $\overline{\mathbf{X}}_{+}$can be written in terms of the electroneutral thermodynamic factor and Poisson overpotential as

$$
\overline{\mathbf{X}}_{+}=\frac{R T}{1-y_{0}}\left[\begin{array}{cc}
\frac{\chi_{0}}{y_{0}}+\frac{\sinh ^{2}(z \phi)}{\cosh (z \phi)} & 2 \tanh (z \phi) \\
2 \tanh (z \phi) & \frac{4}{\cosh (z \phi)}
\end{array}\right]
$$

It is straightforward to verify that the trace and determinant of this matrix are strictly non-negative for all finite $\phi$. Since both its eigenvalues must therefore be positive, this choice of the Hessian is thermodynamically consistent, in the sense that it guarantees every equilibrium composition (including non-neutral compositions) will be thermodynamically stable.

Ultimately, including the functionality $\chi_{\mathrm{e}}\left(y_{0}, \phi\right)$ from equation 121 , then returning to the naive potential $\Phi$ by redimensionalizing $\phi$ with equation 104 and applying equation 102, produces a simpler form of equations 123 ,

$$
\begin{aligned}
d \mu_{0}= & R T \chi_{0} d \ln y_{0}, \\
d \mu_{+}= & R T \chi_{0} d \ln \left(1-y_{0}\right) \\
& +F z\left[1-\sinh \left(\frac{F z \Phi}{R T}\right)\right] d \Phi, \\
d \mu_{-}= & R T \chi_{0} d \ln \left(1-y_{0}\right) \\
& -F z\left[1+\sinh \left(\frac{F z \Phi}{R T}\right)\right] d \Phi .
\end{aligned}
$$

These are similar - but not identical - to the constitutive laws that are commonly used to underpin transport 
models based on dilute-solution theory or Poisson-NernstPlanck theory; both theories involve a foundational assumption that the electrochemical potential of species $i$ varies as $d \mu_{i}=R T d \ln y_{i}+F z_{i} d \Phi$ [22]. The modifications in equation 123 ensure consistency of the Boltzmann equation of state, equation 100, through the terms that involve hyperbolic functions of naive potential; consistency with electroneutral concentrated-solution theory is enabled by allowing $\chi_{0}$ to deviate from 1 . Direct calculation verifies that constitutive laws 123 satisfy the Gibbs-Duhem equation, so this formulation avoids the fundamental difficulty mentioned in the discussion after equation 33 above.

\section{Synopsis}

Electrolytic fluids are defined in part by their obeyance of Faraday's law, equation 22, which demands that species molar contents within a material determine its total charge. The linearity of this law's intensive form, equation 23, makes it straightforward to transform the natural expression of composition, in terms of a set of independent particle fractions defined by equation 4 , into an alternative, neutralizable representation, in which information about the charge state is embodied by a single independent variable. To define a secondary reference state, we specified a particular pair of countercharged species within the material being described, naming them the reference ion and the charging ion.

A neutralizable composition basis can generally be constructed with respect to the two-ion reference state by taking the reference ion's particle fraction to depend on those remaining through the phase rule, equation 5 , and by replacing the charging ion's particle fraction with the molar excess charge. Equation 25 constructs an involutory affine transformation that carries out this basis change. Adoption of the two-ion reference state also ensures that to each neutralizable composition there corresponds a unique electroneutral reference composition, given by equation 86 .

The move to a two-ion secondary reference state produces a new set of thermodynamic potentials, which we call the core electrochemical potentials. These arise naturally as thermodynamic conjugates of the neutralizable composition variables when the first law of thermodynamics is expressed in terms of the molar Gibbs free energy $\bar{G}$, as can be seen from equation 46 . Core potentials have the advantage that just one of their number is conjugate to molar charge. This naive electric potential, defined by equation 28, contains all the information about how an electrolyte's free energy is affected by changes in its charge state. Through equation 88 , it can be partitioned into an electroneutral part and an external Poisson overpotential, which drives charge accumulation.

Core potentials with respect to a two-ion reference state prove to depend explicitly on the electrochemical potentials originally defined by Guggenheim [19]. Molar Gibbs energy obeys the first law of thermodynamics and satisfies an Euler equation: these foundational thermodynamic laws must contain identical information whether they are expressed over the particle-fraction basis, as in equations 8 and 11, or over the neutralizable composition basis, as in equations 46 and 47 . Taking an approach inspired by Kokotov [21], we equated the representations of $\bar{G}$ and $d \bar{G}$ over the two composition bases, then used linear independence arguments to construct the invertible transformations in equations 49 and 50, which express the core potentials as linear combinations of the Guggenheim potentials, or vice versa. Passing through the Guggenheim potentials, one can also transform the core potentials into the quasielectrostatic electrochemical potentials defined by Smyrl and Newman [20], through an invertible linear mapping expressed by the transformations constructed in equations 80 and 81.

Explicitly constructing all of the interconversions between composition bases and among sets of thermodynamic potentials enables the implementation of this work's central objective: the establishment of a parsimonious set of thermodynamic factors to underpin transport models that involve the independent mass-transfer driving forces listed in equation 17. From the subset of independent driving forces, we formed the complete set of mass-transfer driving forces in equation 64 through an algebraic process called Gibbs-Duhem extension, implemented in this case by applying equations 65 and 66 .

There is a direct linear-algebraic relationship, expressed by matrix equation 60 , between the thermodynamic factors for Guggenheim potentials over the particle-fraction basis and the thermodynamic factors for core potentials over the neutralizable composition basis established by the two-ion secondary reference state. This conversion employs a pair of linear transformations: the first, an involution whose matrix representation $\mathbf{T}_{n-1}^{d x \leftrightarrow d y}$ is constructed in equation 27, depends only on the set of equivalent charges, and converts differential changes in the particle fractions into changes in the neutralizable composition; the second, defined in equation 19, depends only on the composition variables.

In an $n$-species electrolyte, all the activity coefficients that appear in the standard Darken-factor matrix defined by equation 91 , depend directly and invertibly on the matrix of core thermodynamic factors $\overline{\mathbf{X}}_{n-1}$, defined by equation 54. Thus the composition-dependent parts of the set of thermodynamic mass-transfer driving forces for an electrolytic fluid can generally be parameterized in a thermodynamically consistent way by defining a set of constitutive laws summarized by the matrix relation

$$
d\left[\mu_{i}+\bar{S}_{i} d T-\bar{V}_{i} d p\right]_{n}=\mathbf{T}_{n}^{\mu \leftrightarrow \mathrm{M}^{n}} \boldsymbol{\Gamma}_{x} \overline{\mathbf{X}}_{n-1} \mathbf{T}_{n-1}^{d x \hookleftarrow d y} d\left[y_{i}\right]_{n-1},
$$

in which the two-ion secondary reference state ensures that every quantity on the right except the matrix of core thermodynamic factors depends in a specific way on the composition variables and the set of equivalent charges ( $c f$. equations 50 and 62). Moreover, Maxwell relations, constraints realized as a matrix symmetry in equation 57 , 
imply that only $\frac{1}{2} n(n-1)$ of the individual core thermodynamic factors that constitute $\overline{\mathbf{X}}_{n-1}$ are independent. The electroneutral-reference-composition dependences of $\frac{1}{2}(n-1)(n-2)$ of these factors can be established directly, producing harmony with the parameterizations of multicomponent electrolytes in Newman's electroneutral concentrated solution theory [22]. The remaining $n-1$ core thermodynamic factors express how an equilibrated electrolyte responds to reversible changes in its charge state at fixed temperature and pressure.

Equation 78, which recasts equation 124 in terms of changing neutralizable composition rather than changing particle fractions, isolates the impact of charge-density variation within a single independent variable, and therefore may be more useful for practical implementations. That constitutive formulation is explicit in the electrochemical potentials, and therefore also necessites ancillary properties that show how the electrochemical potentials depend on pressure and volume. These properties are defined by equation 71, and called partial core volumes and partial core entropies. Although there are $2 n$ such properties, all but two are unambiguously measurable in systems held at constant charge; those remaining separately quantify piezoelectric and thermoelectric responses to changes in the charge state. Conversion between the standard and neutralizable representations of volume and entropy can be performed with equation 72 , which expresses the partial core properties as invertible linear combinations of partial molar volumes and entropies.

Thermodynamic consistency requires that the set of electrical thermodynamic factors and the equation of state that relates charge density to electric potential are mutually dependent. We outline two routes by which this connection can be established. By assuming that the core thermodynamic factors are consistent with the ideal-solution approximation - namely, that all the species activity coefficients over the particle-fraction basis are constant with respect to composition - we produced thermodynamically consistent equations of state for naive potential and charge density, equations 97 and 98, respectively. Alternatively, by adopting the Poisson-Boltzmann equations of state for naive potential and charge density, given respectively by equations 102 and 100, we showed that the electrical core thermodynamic factors must have the functionality given in equation 103.

Both approaches to the electrical colligative properties begged further questions. The ionic strength that derives from the ideal-solution approximation, given by equation 99, does not match Lewis and Randall's familiar definition [56] and depends only on the reference and charging ions' electroneutral particle fractions. On the other hand, the electrical thermodynamic factors mandated by PoissonBoltzmann theory seemed to imply through equation 59 that the Gibbs function can cease to be convex if the Poisson overpotential grows sufficiently large.

We considered in detail the example of an isother- mal, isobaric binary solution of a symmetric salt in a neutral solvent, which we assumed to be further constrained such that its excess charge relates to the external potential that induces non-neutrality through the PoissonBoltzmann equation of state (equation 100). This analysis showed that it is indeed possible to choose the composition dependence of the core-potential Hessian in such a way that convexity of the Gibbs function is retained in all electrical states.

Ultimately, we produced a thermodynamically consistent set of constitutive equations for species electrochemical potentials in the binary, symmetric Poisson-Boltzmann electrolyte. These are given by equations 123, which are cast in terms of the naive potential $\Phi$, in turn related to the external potential through equation 102. As well as ensuring thermodynamic stability in non-neutral states, the new system of constitutive laws provided by equations 102 , 104-106, and 123 naturally incorporates chemical nonideality through a neutral thermodynamic factor that can be measured in traditional ways, as well as ensuring consistency with the Gibbs-Duhem equation should electroneutrality be violated.

Further work is clearly needed to establish uncontroversial and thermodynamically rigorous equations of state that can be used to relate local charge density to local voltage for other nonideal, non-neutral, multicomponent electrochemical solutions. The two-ion secondary reference state and the core potential formalism outlined here provide a thermodynamic framework consistent with concentrated solution theory, within which it should be possible to develop constitutive formulations that can be applied to additional practically relevant systems, such as the asymmetric-valence Poisson-Boltzmann binary electrolyte, the three-ion ionic liquid, and the two-solvent lithiumion electrolyte.

\section{Acknowledgments}

This work was supported by the Faraday Institution, subawards FIRG003 and FIRG007 under EPSRC grant no. EP/S003053/1, and the ISCF Materials Research Hub for Energy Conversion, Capture, and Storage (M-RHEX), grant no. EP/R023581/1. CWM would like to thank A van-Brunt for his proof that the charge-potential relationship from Poisson-Boltzmann theory is invertible.

\section{References}

[1] E. A. Guggenheim, Thermodynamics: an Advanced Treatment for Chemists and Physicists, $5^{\text {th }}$ Ed., North-Holland Publishing Company, Amsterdam, 1967.

[2] F. Malatesta, Comment on the individual ion activities of $\mathrm{Na}^{+}$ and $\mathrm{Cl}^{-}$by Arce, Wilczek-Vera and Vera, Chemical Engineering Science 65 (2010) 675-679.

[3] B. Pethica, Are electrostatic potentials between regions of different chemical composition measurable? The Gibbs-Guggenheim principle reconsidered, extended and its consequence revisited, Physical Chemistry Chemical Physics 9 (2007) 6253-6262. 
[4] R. Smith, M. Bazant, Multiphase porous electrode theory, Journal of the Electrochemical Society 164 (2017) E3291-E3310.

[5] P. J. W. Debye, E. Hückel, On the theory of electrolytes. I. Freezing point depression and related phenomena, trans. from Physikalische Zeitschrift 24 (1923) 185-206, in: The Collected Papers of Peter J. W. Debye, Interscience Publishers, Inc., London, 1954, pp. 217-263.

[6] D. L. Chapman, A contribution to the theory of electrocapillarity, Philosophical Magazine 25 (1913) 475-481.

[7] O. Stern, Zur Theorie der elektrolytischen Doppelschicht, Zeitschrift für Elektrochemie 30 (1924) 508-516.

[8] A. A. Kornyshev, M. A. Vorotyntsev, Nonlocal electrostatic approach to the double layer and adsorption at the electrodeelectrolyte interface, Surface Science 101 (1980) 23-48.

[9] M. Planck, Ueber die Potentialdifferenz zwischen zwei verdünten Lösungen binärer Electrolyte, Annalen der Physik und Chemie 40 (1890) 561-576.

[10] V. G. Levich, Physicochemical Hydrodynamics, Prentice Hall, Englewood Cliffs, New Jersey, 1962.

[11] H. Cohen, J. W. Cooley, The numerical solution of the timedependent Nernst-Planck equations, Biophysical Journal 5 (1965) 145-162.

[12] J. Newman, The polarized diffuse double layer, Transactions of the Faraday Society 61 (1965) 2157-2163.

[13] E. J. W. Verwey, J. T. G. Overbeek, Theory of the Stability of Lyophobic Colloids, Elsevier, Amsterdam, 1948.

[14] J. R. Melcher, G. I. Taylor, Electrohydrodynamics: a review of the role of interfacial shear stresses, Annual Review of Fluid Mechanics 1 (1969) 111-146.

[15] D. Saville, Electrohydrodynamics: The Taylor-Melcher leaky dielectric model, Annual Review of Fluid Mechanics 29 (1997) $27-64$.

[16] M. Z. Bazant, K. Thornton, A. Ajdari, Diffuse-charge dynamics in electrochemical systems, Physical Review E 70 (2004) 021506 .

[17] C. W. Monroe, M. Urbakh, A. A. Kornyshev, The distinctive electrowetting properties of ITIES, Journal of Physics: Condensed Matter 19 (2007) 375113.

[18] A. Hemmatifar, A. Ramachandran, K. Liu, D. Oyarzun, M. Bazant, J. Santiago, Thermodynamics of ion separation by electrosorption, Environmental Science and Technology 52 (2018) 10196-10204.

[19] E. A. Guggenheim, The conceptions of electrical potential difference between two phases and the individual activities of ions, Journal of Physical Chemistry 33 (1929) 842-849.

[20] W. H. Smyrl, J. Newman, Potentials of cells with liquid junctions, Journal of Physical Chemistry 72 (1968) 4660-4671.

[21] Y. Kokotov, Electrochemical and twin chemical potentials as thermodynamic driving forces, Solvent Extraction and Ion Exchange 17 (1999) 1083-1131.

[22] J. Newman, K. E. Thomas-Alyea, Electrochemical Systems, $3^{\text {rd }}$ Edition, John Wiley and Sons, Inc., Hoboken, New Jersey, 2004.

[23] J. Garrido, J. Manzanares, Observable electric potential and electrostatic potential in electrochemical systems, The Journal of Physical Chemistry B 104 (2000) 658-662.

[24] M. Ganser, F. Hildebrand, M. Kamlah, R. McMeeking, A finite strain electro-chemo-mechanical theory for ion transport with application to binary solid electrolytes, Journal of the Mechanics and Physics of Solids 125 (2019) 681-713.

[25] A. Kornyshev, Double-layer in ionic liquids: Paradigm change?, The Journal of Physical Chemistry B 111 (2007) 5545-5557.

[26] A. Levy, J. de Souza, M. Bazant, Breakdown of electroneutrality in nanopores, Journal of Colloid and Interface Science 579 (2020) 162-176.

[27] M. Matse, P. Berg, M. Eikerling, Asymmetric double-layer charging in a cylindrical nanowire under closed confinement, The Journal of Chemical Physics 152 (2020) 084103

[28] K. Kobayashi, T. Suzuki, Theoretical modelling of electrode overvoltage for an all-solid-state electrochemical device, Japanese Journal of Applied Physics 59 (2020) SIIG04.

[29] M. Hatlo, R. V. Roij, L. Lue, The electric double layer at high surface potentials: The influence of excess ion polarisability, Europhysics Letters 97 (2012) 28010.

30] S. Braun, C. Yada, A. Latz, Thermodynamically consistent model for space-charge-layer formation in a solid electrolyte, Journal of Physical Chemistry C 119 (2015) 22281-22288.

[31] A. Latz, J. Zausch, Thermodynamic consistent transport theory of Li-ion batteries, Journal of Power Sources 196 (2011) 32963302.

[32] P. Peters, R. van Roij, M. Bazant, P. Biesheuvel, Analysis of electrolyte transport through charged nanopores, Physical Review E 93 (2016) 053108.

[33] M. Camci, B. Ulgut, C. Kocabas, S. Suzer, In situ XPS reveals voltage driven asymmetric ion movement of an ionic liquid through the pores of a multilayer graphene electrode, The Journal of Physical Chemistry C 122 (2018) 11883-11889.

[34] X. Luo, Y. Xing, Y. Ling, A. Kleinhammes, Y. Wu, Electroneutrality breakdown and specific ion effects in nano confined aqueous electrolytes observed by NMR, Nature Communications 6 (2015) 6358.

[35] Y. Nomura, K. Yamamoto, T. Hirayama, S. Ouchi, E. Igaki, K. Saitoh, Direct observation of a Li-ionic space-charge layer formed at an electrode/solid-electrolyte interface, Angewandte Chemie 58 (2018) 5292-5296.

[36] A. M. Smith, A. A. Lee, S. Perkin, The electrostatic screening length in concentrated electrolytes increases with concentration, The Journal of Physical Chemistry Letters 7 (2016) 2157-2163.

[37] S. Rivera, T. Sørensen, Grand canonical ensemble Monte Carlo simulations of Donnan potentials, nonelectroneutrality, activity coefficients and excess energy in spherical charged or uncharged pores with restricted primitive model electrolytes, Molecular Simulation 13 (1994) 115-160.

[38] M. Swift, Y. Qi, First-principles prediction of potentials and space-charge layers in all-solid-state batteries, Physical Review Letters 122 (2019) 167701

[39] P. Vágner, C. Guhlke, V. Miloš, R. Müller, J. Fuhrmann, A continuum model for yttria-stabilized zirconia incorporating triple phase boundary, lattice structure and immobile oxide ions, Journal of Solid State Electrochemistry 23 (2019) 2907-2926.

[40] W. Morf, E. Pretsch, N. de Rooij, Theoretical treatment and numerical simulation of potential and concentration profiles in extremely thin non-electroneutral membranes used for ionselective electrodes, Journal of Electroanalytical Chemistry 641 (2010) 45-56.

41] J. Liu, B. Eisenberg, Poisson-Nernst-Planck-Fermi theory for modelling biological ion channels, The Journal of Chemical Physics 141 (2014) 22D532.

[42] E. Dickinson, J. Limon-Petersen, R. Compton, The electroneutrality approximation in electrochemistry, Journal of Solid State Electrochemistry 15 (2011) 1335-1345.

[43] L. Onsager, Theories and problems of liquid diffusion, Annals of the New York Academy of Sciences 46 (1945) 241-265.

[44] P. Goyal, C. W. Monroe, New foundations of Newman's theory for solid electrolytes: Thermodynamics and transient balances, Journal of the Electrochemical Society 164 (2017) E3647-E3660.

[45] J. Hirschfelder, C. Curtiss, R. Bird, The Molecular Theory of Gases and Liquids, John Wiley \& Sons, New York, 1954.

[46] S. R. de Groot, Thermodynamics of Irreversible Processes, Interscience Publishers, New York, 1951

[47] S. R. de Groot, P. Mazur, Non-Equilibrium Thermodynamics, North-Holland, Amsterdam, 1962

[48] C. W. Monroe, J. Newman, Onsager reciprocal relations for Stefan-Maxwell diffusion, Industrial and Engineering Chemistry Research 45 (2006) 5361-5367.

[49] L. S. Darken, Diffusion, mobility and their interrelation through free energy in binary metallic systems, Transactions of the AIME 175 (1948) 184-201.

[50] C. W. Monroe, D. R. Wheeler, J. Newman, Nonequilibrium linear response theory: Application to Onsager-Stefan-Maxwell diffusion, Industrial \& Engineering Chemistry Research 54 (2015) 4460-4467.

[51] C. W. Monroe, J. Newman, Onsager's shortcut to proper fluxes 
and forces, Chemical Engineering Science 64 (2009) 4804-4809.

[52] C. Monroe, C. Delacourt, Continuum transport laws for locally non-neutral concentrated electrolytes, Electrochimica Acta 114 (2013) 649-657.

[53] A. Bizeray, D. Howey, C. Monroe, Resolving a discrepancy in diffusion potentials, with a case study for Li-ion batteries, Electrochimica Acta 114 (2013) 649-657.

[54] R. A. Robinson, R. H. Stokes, Electrolyte Solutions, $2^{\text {nd }}$ Ed. (Revised), Butterworths, London, U.K., 1968.

[55] J. J. Sylvester, A demonstration of the theorem that every homogeneous quadratic polynomial is reducible by real orthogonal substitution to the form of a sum of positive and negative squares, Philosophical Magazine 4 (1852) 138-142.

[56] G. N. Lewis, M. Randall, The activity coefficient of strong electrolytes, Journal of the American Chemical Society 43 (1921) 1112-1154.

[57] E. A. Guggenheim, The specific thermodynamic properties of aqueous solutions of strong electrolytes, The London, Edinburgh, and Dublin Philosophical Magazine and Journal of Science 19 (1935) 588-643.

[58] K. S. Pitzer, J. J. Kim, Thermodynamics of electrolytes. IV. Activity and osmotic coefficients for mixed electrolytes, Journal of the American Chemical Society 96 (1974) 5701-5707.

[59] J. Liu, C. W. Monroe, Solute-volume effects in electrolyte transport, Electrochimica Acta 135 (2014) 447-460.

[60] T. W. Chapman, J. Newman, A Compilation of Selected Thermodynamic and Transport Properties of Binary Electrolytes in Aqueous Solution, Technical Report UCRL-17767, University of California, Lawrence Radiation Laboratory, 1968.

[61] T. Hou, C. W. Monroe, Composition-dependent thermodynamic and mass-transport characterization of lithium hexafluorophosphate in propylene carbonate, Electrochimica Acta 332 (2020) 135085.

[62] A. A. Wang, T. Hou, M. Karanjavala, C. W. Monroe, Shiftingreference concentration cells to refine composition-dependent transport characterization of binary lithium-ion electrolytes, Electrochimica Acta 358 (2020) 136688.

[63] J. Landesfeind, H. A. Gasteiger, Temperature and concentration dependence of the ionic transport properties of lithium-ion battery electrolytes, Journal of the Electrochemical Society 166 (2019) A3079-A3097.

\section{Appendix A. Derivation of the intensive first law}

Start by substituting equation 4 into equation 1 ,

$$
d G=-S d T+V d p+\sum_{i=1}^{n} \mu_{i} d\left(n_{\mathrm{T}} y_{i}\right)
$$

Use the phase rule, equation 5 , to show that

$$
\begin{aligned}
d G= & -S d T+V d p+\mu_{n} d\left[n_{\mathrm{T}}\left(1-\sum_{i=1}^{n-1} y_{i}\right)\right] \\
& +\sum_{i=1}^{n-1} \mu_{i} d\left(n_{\mathrm{T}} y_{i}\right),
\end{aligned}
$$

expand the differentials and rearrange, yielding

$$
\begin{aligned}
d G= & -S d T+V d p+n_{\mathrm{T}} \sum_{i=1}^{n-1}\left(\mu_{i}-\mu_{n}\right) d y_{i} \\
& +d n_{\mathrm{T}} \sum_{i=1}^{n} \mu_{i} y_{i},
\end{aligned}
$$

then bring in equation 8 and rearrange again to get

$$
d G-\bar{G} d n_{\mathrm{T}}=-S d T+V d p+n_{\mathrm{T}} \sum_{i=1}^{n-1}\left(\mu_{i}-\mu_{n}\right) d y_{i} .
$$

The molar Gibbs energy relates to $G$ through $G=\bar{G} n_{\mathrm{T}}$ by definition, so expansion of the differential in $d G$ gives

$$
n_{\mathrm{T}} d \bar{G}=-S d T+V d p+n_{\mathrm{T}} \sum_{i=1}^{n-1}\left(\mu_{i}-\mu_{n}\right) d y_{i} .
$$

Introduction of molar quantities through $S=\bar{S} n_{\mathrm{T}}$ and $V=$ $\bar{V} n_{\mathrm{T}}$, followed by incorporation of the definition $c_{\mathrm{T}}=1 / \bar{V}$, produces equation 11 .

\section{Appendix B. The $n^{\text {th }}$ neutralizable composition variable}

Augment the $(n-1)$-tuple $\left\{x_{i}\right\}_{n-1}$ by adding entry $x_{n}$ such that it equals $y_{n}$, bearing in mind that one can express $x_{n}=y_{n}$ either in terms of independent particle fractions or the neutralizable composition,

$$
\begin{aligned}
x_{n} & =1-\sum_{i=1}^{n-1} y_{i} \\
& =-\frac{z_{n-1}}{z_{n}-z_{n-1}}+\sum_{i=1}^{n-2}\left(\frac{z_{n-1}-z_{i}}{z_{n}-z_{n-1}}\right) x_{i}+x_{n-1} .
\end{aligned}
$$

Whenever $x_{n}=y_{n}>0$, the map $\left\{y_{i}\right\}_{n} \leftrightarrow\left\{x_{i}\right\}_{n}$ can be written in a matrix representation

$$
\left[x_{i}\right]_{n}=\mathbf{T}_{n}^{x \leftrightarrow y}\left[y_{i}\right]_{n} \quad \text { or } \quad\left[y_{i}\right]_{n}=\mathbf{T}_{n}^{y \leftrightarrow x}\left[x_{i}\right]_{n},
$$

wherein $\mathbf{T}_{n}^{x \leftrightarrow y}$ is an invertible matrix dependent only on $y_{n}\left(\right.$ or $\left.x_{n}\right)$, with entries

$$
\begin{aligned}
\left(\mathbf{T}_{n}^{x \leftrightarrow y}\right)_{i j}= & \left(1-\delta_{i(n-1)}\right) \delta_{i j}-\delta_{i(n-1)} \times \\
& {\left[\left(\frac{z_{n}-z_{j}}{z_{n}-z_{n-1}}\right)-\frac{\delta_{j n} z_{n}}{\left(z_{n}-z_{n-1}\right) y_{n}}\right] . }
\end{aligned}
$$

Note that $\mathbf{T}_{n}^{x \leftrightarrow y}$ is involutory, so that $\mathbf{T}_{n}^{x \leftrightarrow y}=\mathbf{T}_{n}^{y \leftrightarrow x}$. Despite the nonlinear and nonaffine nature of $\mathbf{T}_{n}^{x \leftrightarrow y}$, matrix equation B.2 helps to implement basis changes in governing equations that contain all $n$ of the particle fractions. In numerical implementations, this also may be useful to convert on the fly between the sets $\left\{x_{i}\right\}_{n}$ and $\left\{y_{i}\right\}_{n}$.

\section{Appendix C. Partial molar properties from the molar Gibbs function}

The connection between core potentials and partial molar volumes is ambiguous because the composition basis under $G\left(T, p,\left\{n_{i}\right\}_{n}\right)$ differs in both dimension and extensivity properties from the basis under $\bar{G}\left(T, p,\left\{y_{i}\right\}_{n-2}, \overline{\mathrm{P}}\right)$. Nevertheless, the pressure differentiation of equation 47 to determine a volumetric equation of state is indeed a sound strategy, as follows.

Equation 2 and the definition $c_{\mathrm{T}}=n_{\mathrm{T}} / V$ can be combined with the result obtained when differentiating equation 41 with $p$ at constant temperature, chemical composition, and electrical state to show that

$$
\frac{1}{n_{\mathrm{T}}}\left(\frac{\partial G}{\partial p}\right)_{T, n_{i}}=\left(\frac{\partial \bar{G}}{\partial p}\right)_{T, y_{i<n-1}, \overline{\mathrm{P}}} .
$$


On the right, replace $\bar{G}$ with equation 8 , noting that equation 5 requires $y_{n}\left(\left\{y_{i}\right\}_{n-1}\right)$; on the left, observe that the function $G\left(T, p,\left\{n_{i}\right\}_{n}\right)$ satisfies the Euler equation for the grand canonical ensemble, equation 7 , and that $n_{i}$ is independent of $p$ for all $i$. Rearrangement with equations 4, 5, and 25 then yields

$$
\sum_{i=1}^{n}\left[\left(\frac{\partial \mu_{i}}{\partial p}\right)_{T, n_{i}}-\left(\frac{\partial \mu_{i}}{\partial p}\right)_{T, y_{i<n-1}, \overline{\mathrm{P}}}\right] n_{i}=0 .
$$

Since $\left\{n_{i}\right\}_{n}$ is a basis, its elements are linearly independent. Thus the bracketed term corresponding to each index $i$ necessarily vanishes, demonstrating equivalence of the two partial derivatives in equation 67 .

\section{Appendix D. Bulk modulus and thermal expan- sion coefficient}

Many liquids experience volume strain when subjected to changes in temperature or pressure, but this possible functionality of $c_{\mathrm{T}}$ is hidden within the partial molar properties defined so far. Since $\left\{y_{i}\right\}_{n-1}$ and $\left\{x_{i}\right\}_{n-1}$ are independent of $T$ and $p$, volumetric state equations 9 and 73 show that any reversible tendency of a material to thermally expand, or to compress under pressure, must be reflected by $T$ and $p$ dependences of the properties $\left\{\bar{V}_{i}\right\}_{n}$ or $\left\{\overline{\mathrm{v}}_{i}^{n}\right\}_{n}$.

Considering that $V\left(T, p,\left\{n_{i}\right\}_{n}\right)$ (see equation 2 et seq.), that $V$ is extensive, and that $c_{\mathrm{T}}=n_{\mathrm{T}} / V$ by definition, one can differentially expand $V / n_{\mathrm{T}}$, rearrange using Euler equation 9 , change from $\left\{d y_{i}\right\}_{n-1}$ to $\left\{d x_{i}\right\}_{n-1}$, and introduce the first $n-1$ partial core volumes, $\left\{\overline{\mathrm{v}}_{i}^{n}\right\}_{n-1}$, to get

$$
d\left(\frac{1}{c_{\mathrm{T}}}\right)=\frac{\alpha_{V}}{c_{\mathrm{T}}} d T-\frac{1}{c_{\mathrm{T}} K} d p+\sum_{i=1}^{n-1} \overline{\mathrm{v}}_{i}^{n} d x_{i}
$$

which makes the $T$ and $p$ dependences of $c_{\mathrm{T}}$ explicit through the volumetric coefficient of thermal expansion $\alpha_{V}$ and the bulk modulus (inverse isothermal compressibility) $K$, conventionally defined as

$$
\alpha_{V}=\frac{1}{V}\left(\frac{\partial V}{\partial T}\right)_{p, n_{i}} \text { and } \quad \frac{1}{K}=-\frac{1}{V}\left(\frac{\partial V}{\partial p}\right)_{T, n_{i}} .
$$

Equation D.1 shows that each entry in the subset $\left\{\overline{\mathrm{v}}_{i}^{n}\right\}_{n-1}$ satisfies equation 74 .

To complete a practical parameterization using equations 74 , one also needs expressions for $\overline{\mathrm{v}}_{n}^{n}$ and $\overline{\mathrm{s}}_{n}^{n}$. Insert equation 73 into equation D.1, producing

$$
d \overline{\mathrm{v}}_{n}^{n}=\frac{\alpha_{V}}{c_{\mathrm{T}}} d T-\frac{1}{c_{\mathrm{T}} K} d p-\sum_{i=1}^{n-1} x_{i} d \overline{\mathrm{v}}_{i}^{n} .
$$

Given the properties on the right as functions of $T, p$, and $\left\{x_{i}\right\}_{n-1}$, this can be path integrated from any point where $c_{\mathrm{T}}$ is known to set $\overline{\mathrm{v}}_{n}^{n}\left(T, p,\left\{x_{i}\right\}_{n-1}\right)$. Similarly,

$$
d \overline{\mathrm{s}}_{n}^{n}=\frac{\bar{C}_{p}}{T} d T+\frac{\alpha_{V}}{c_{\mathrm{T}}} d p-\sum_{i=1}^{n-1} x_{i} d \overline{\mathrm{s}}_{i}^{n},
$$

where $\bar{C}_{p}$ is the molar constant-pressure heat capacity, can set $\overline{\mathrm{s}}_{n}^{n}\left(T, p,\left\{x_{i}\right\}_{n-1}\right)$ relative to a suitable reference point.

\section{Appendix E. Direct transformations of potential derivatives}

Guggenheim potentials and core potentials contain identical information. If equations 77 and 78 are both true, then it must be the case that

$$
\mathbf{T}_{n}^{\mu \leftrightarrow \mathrm{M}^{n}} \boldsymbol{\Gamma}_{x} \overline{\mathbf{X}}_{n-1} \mathbf{T}_{n-1}^{d x \leftrightarrow d y}=\mathbf{y}_{n}^{-1} \mathbf{Q} .
$$

Insertion of equations 65 and 60 shows that the equations are consistent if and only if

$$
\mathbf{T}_{n}^{\mu \leftrightarrow \mathrm{M}^{n}} \boldsymbol{\Gamma}_{x}=\mathbf{y}_{n}^{-1} \boldsymbol{\Gamma}_{1} \mathbf{Y}_{n-1}^{-1}\left(\mathbf{T}_{n-1}^{d x \leftrightarrow d y}\right)^{\mathrm{T}}
$$

Direct expansion of the matrix product on the left side of this equation yields the indicial form

$$
\left(\mathbf{T}_{n}^{\mu \leftrightarrow \mathrm{M}^{n}} \boldsymbol{\Gamma}_{x}\right)_{i j}=\delta_{i j}\left(1-\delta_{(n-1) j}\right)-x_{j}+\frac{z_{i} \delta_{(n-1) j}}{z_{n}-z_{n-1}},
$$

which matches the indicial form of the matrix product on the right.

A truncated version of the matrix in equation E.3 can be seen as a transformation that sends changes in the independently variable core potentials into changes in the Guggenheim potentials. In this transformation, which can be notated as

$$
[d \mu]_{n-1}=\mathbf{T}_{n-1}^{d \mu \leftrightarrow d \mathrm{M}^{n}}\left[d \mathrm{M}^{n}\right]_{n-1},
$$

the $(n-1) \times(n-1)$ matrix $\mathbf{T}_{n}^{d \mu \leftrightarrow d \mathrm{M}^{n}}$ has entries

$$
\left(\mathbf{T}_{n}^{d \mu \leftrightarrow d \mathrm{M}^{n}}\right)_{i j}=\left(\mathbf{T}_{n}^{\mu \leftrightarrow \mathrm{M}^{n}} \boldsymbol{\Gamma}_{x}\right)_{i j}
$$

for $i, j<n$. One can invert this to show that the formula

$$
\begin{aligned}
\left(\mathbf{T}_{n}^{d \mathrm{M}^{n} \leftrightarrow d \mu}\right)_{i j}= & \left(\left(\mathbf{T}_{n}^{d \mu \leftrightarrow d \mathrm{M}^{n}}\right)^{-1}\right)_{i j} \\
= & \delta_{i j}-\left(\frac{z_{n}-z_{i}}{z_{n}-z_{n-1}}+\delta_{i(n-1)}\right) \delta_{(n-1) j} \\
& +\left(\frac{z_{i}-z_{n-1}}{z_{n}-z_{n-1}}-\delta_{i(n-1)}\right) \frac{y_{j}}{y_{n}}
\end{aligned}
$$

yields the transformation from $\left\{d \mu_{i}\right\}_{n-1}$ into $\left\{d \mathrm{M}_{i}^{n}\right\}_{n-1}$. 